\title{
The Effects of Substituents on the Stability of Phosphoranyl Radicals
}

\author{
Jennifer L. Hodgson and Michelle L. Coote*
}

Research School of Chemistry, Australian National University, Canberra, ACT 0200, Australia

\section{SUPPORTING INFORMATION}

*Email address for correspondence, email: mcoote@rsc.anu.edu.au 


\section{Table S1. B3-LYP/6-31G(d) Optimized Geometries}

\section{$\cdot P\left(\mathrm{CH}_{3}\right)_{4}(1)$}

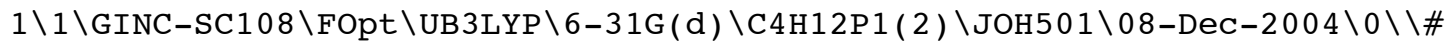

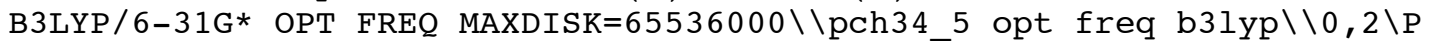
$, 0.0 ., 0.3115948418 \backslash \mathrm{C}, 1.9779003667,0 ., 0.6232678829 \backslash \mathrm{C},-1.9779003667,0$. , $0.6232678829 \backslash \mathrm{C}, 0 .,-1.4676715436,-0.8330835307 \backslash \mathrm{C}, 0 ., 1.4676715436,-0.833$ $0835307 \backslash \mathrm{H}, 2.5484816755,0$. $,-0.3158410104 \backslash \mathrm{H},-2.5484816755,0 .,-0.31584101$ $04 \backslash \mathrm{H}, 2.2304646838,-0.8897497538,1.2084273618 \backslash \mathrm{H}, 2.2304646838,0.88974975$ $38,1.2084273618 \backslash \mathrm{H},-2.2304646838,0.8897497538,1.2084273618 \backslash \mathrm{H},-2.2304646$ $838,-0.8897497538,1.2084273618 \backslash \mathrm{H}, 0$. $,-2.3908292027,-0.2426842864 \backslash \mathrm{H}, 0$. , 2 $.3908292027,-0.2426842864 \backslash \mathrm{H},-0.8908674176,-1.4713549552,-1.4681984268 \backslash$ $\mathrm{H}, 0.8908674176,-1.4713549552,-1.4681984268 \backslash \mathrm{H}, 0.8908674176,1.4713549552$ $,-1.4681984268 \backslash \mathrm{H},-0.8908674176,1.4713549552,-1.4681984268 \backslash \backslash$ Version=DEC $-\mathrm{AXP}-\mathrm{OSF} / 1-\mathrm{G} 03 \mathrm{RevB} .03 \backslash \mathrm{State}=2-\mathrm{A} 1 \backslash \mathrm{HF}=-500.9355065 \backslash \mathrm{S} 2=0.755056 \backslash \mathrm{S} 2-1=0 . \backslash \mathrm{S}$ $2 A=0.750019 \backslash \mathrm{RMSD}=5.703 e-09 \backslash \mathrm{RMSF}=2.969 \mathrm{e}-05 \backslash \mathrm{Dipole}=0,0 .,-0.384381 \backslash \mathrm{PG}=\mathrm{C} 0$ $2 \mathrm{~V}\left[\mathrm{C} 2(\mathrm{P} 1), \mathrm{SGV}(\mathrm{C} 2 \mathrm{H} 2), \mathrm{SGV}^{\prime}(\mathrm{C} 2 \mathrm{H} 2), \mathrm{X}(\mathrm{H} 8)\right] \backslash \backslash @$

\section{$\mathrm{HP}\left(\mathrm{CH}_{3}\right)_{4}$}

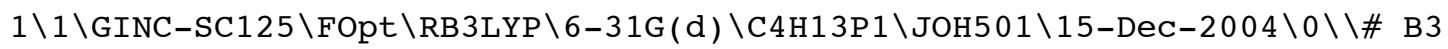

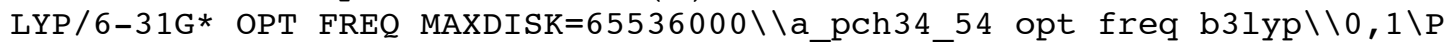
$, 0.0 ., 0.2700472557 \backslash \mathrm{H}, 0 \ldots, 0.1 .717574262 \overline{1} \backslash \mathrm{C}, 1 . \overline{9} 444659545,0$. 0.423960071 $4 \backslash \mathrm{C},-1.9444659545,0 \ldots, 0.4239600714 \backslash \mathrm{C}, 0 \ldots,-1.5911746862,-0.7069446721 \backslash \mathrm{C}, 0$ . , $1.5911746862,-0.7069446721 \backslash \mathrm{H}, 2.4814229208,0$. , $-0.5343506787 \backslash \mathrm{H},-2.4814$ $229208,0,-0.5343506787 \backslash \mathrm{H}, 2.2602194686,-0.8847334606,0.9946086449 \backslash \mathrm{H}, 2$. $2602194686,0.8847334606,0.9946086449 \backslash \mathrm{H},-2.2602194686,0.8847334606,0.99$ $46086449 \backslash \mathrm{H},-2.2602194686,-0.8847334606,0.9946086449 \backslash \mathrm{H}, 0 .,-2.4222508568$ $, 0.0077217144 \backslash \mathrm{H}, 0.2 .4222508568,0.0077217144 \backslash \mathrm{H},-0.8974664187,-1.674787$ $6761,-1.3244111349 \backslash \mathrm{H}, 0.8974664187,-1.6747876761,-1.3244111349 \backslash \mathrm{H}, 0.8974$ $664187,1.6747876761,-1.3244111349 \backslash \mathrm{H},-0.8974664187,1.6747876761,-1.3244$ $111349 \backslash \backslash$ Version=DEC-AXP-OSF $/ 1-$ G03RevB . 03 \State $=1-A 1 \backslash \mathrm{HF}=-501.5386144 \backslash \mathrm{RM}$ $\mathrm{SD}=5.221 \mathrm{e}-09 \backslash \mathrm{RMSF}=1.887 \mathrm{e}-05 \backslash \mathrm{Dipole}=0,0,,-0.3123171 \backslash \mathrm{PG}=\mathrm{C} 02 \mathrm{~V} \quad[\mathrm{C} 2(\mathrm{P} 1 \mathrm{H} 1)$, $\left.\mathrm{SGV}(\mathrm{C} 2 \mathrm{H} 2), \mathrm{SGV}^{\prime}(\mathrm{C} 2 \mathrm{H} 2), \mathrm{X}(\mathrm{H} 8)\right] \backslash \backslash @$

\section{Axial $\cdot \mathrm{P}\left(\mathrm{CH}_{3}\right)_{3} \mathrm{SCH}_{3}(2)$}

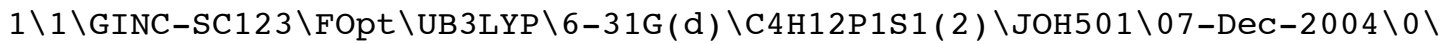
\\# B3LYP/6-31G* OPT FREQ MAXDISK=134217728\\pme3sch3_ax2 opt freq b31y $\mathrm{p} \backslash \backslash 0,2 \backslash \mathrm{S},-1.6892453881,0.8959415254,-0.4716064264 \backslash \mathrm{P}, \overline{0} .688127042,-0.190$ $0068524,0.2853298364 \backslash \mathrm{C}, 1.5498682376,1.4468920022,0.2078980152 \backslash \mathrm{C}, 1.7413$ $197763,-1.0782182442,1.5551412346 \backslash \mathrm{C}, 1.252189797,-1.0035109571,-1.28223$ $33444 \backslash \mathrm{H}, 0.9250422197,-2.0483298262,-1.2945679871 \backslash \mathrm{H}, 2.3434937537,-0.974$ $1317631,-1.3863770509 \backslash \mathrm{H}, 0.7906539201,-0.4916673873,-2.1308368508 \backslash \mathrm{H}, 1.4$ $285029131,-2.1251396384,1.6221123252 \backslash \mathrm{H}, 1.5938503698,-0.6172839001,2.53$ $70190047 \backslash \mathrm{H}, 2.8090958235,-1.042123899,1.3032919286 \backslash \mathrm{H}, 2.627536912,1.3262$ $256656,0.0491020971 \backslash \mathrm{H}, 1.1302935248,2.0422307444,-0.6077846704 \backslash \mathrm{H}, 1.3852$ $326587,1.9912313068,1.1429658109 \backslash \mathrm{C},-2.7626300162,-0.5431003294,-0.1179$ $117639 \backslash \mathrm{H},-3.7881716892,-0.2569820332,-0.3738368837 \backslash \mathrm{H},-2.488488224,-1.4$ $169435277,-0.7161944283 \backslash \mathrm{H},-2.7355083715,-0.8044221908,0.9434971339 \backslash \backslash \mathrm{Ve}$ rsion $=\mathrm{DEC}-\mathrm{AXP}-\mathrm{OSF} / 1-\mathrm{G} 03 \mathrm{RevB} .03 \backslash \mathrm{State}=2-\mathrm{A} \backslash \mathrm{HF}=-899.1679327 \backslash \mathrm{S} 2=0.755196 \backslash \mathrm{S}$ $2-1=0 . \backslash S 2 A=0.750014 \backslash \mathrm{RMSD}=3.485 \mathrm{e}-09 \backslash \mathrm{RMSF}=3.046 \mathrm{e}-06 \backslash \mathrm{Dipole}=1.1242204,-0$. $7882041,0.2777739 \backslash \mathrm{PG}=\mathrm{C} 01 \quad[\mathrm{X}(\mathrm{C} 4 \mathrm{H} 12 \mathrm{P} 1 \mathrm{~S} 1)] \backslash \backslash @$

\section{Axial $\mathrm{HP}\left(\mathrm{CH}_{3}\right)_{3} \mathrm{SCH}_{3}$}

$1 \backslash 1 \backslash G I N C-S C 31 \backslash F O p t \backslash R B 3 L Y P \backslash 6-31 G(d) \backslash C 4 H 13 P 1 S 1 \backslash J O H 501 \backslash 14-D e C-2004 \backslash 0 \backslash \backslash \# B$ 3LYP/6-31G* OPT FREQ MAXDISK=65536000\\a_pme3sch3_ax2 opt freq b3lyp $\backslash \backslash$ 
$0,1 \backslash \mathrm{S},-1.75173029,0.179242632,-0.2322394394 \backslash \mathrm{P}, 0.6897553746,0.077690516$ $4,0.1966866427 \backslash \mathrm{C}, 0.6098913466,1.9186713726,0.3276708279 \backslash \mathrm{C}, 2.4689461386$ $,-0.1022245001,0.8326498138 \backslash \mathrm{C}, 0.9686086919,-0.5973656383,-1.4961001969$ $\backslash \mathrm{H}, 0.5448423842,-1.6043269046,-1.5384312306 \backslash \mathrm{H}, 2.0351315794,-0.63857020$ $51,-1.7404901888 \backslash \mathrm{H}, 0.4217350582,0.0180441012,-2.2118553352 \backslash \mathrm{H}, 2.7525240$ $645,-1.16190909,0.837449038 \backslash \mathrm{H}, 2.5381905594,0.2654236452,1.8640609719 \backslash \mathrm{H}$ ,3.1949936716,0.4474028417,0.2223955513\H, 1.6100849505,2.3570527666, 0 . $2598311101 \backslash \mathrm{H},-0.0411819135,2.3134391987,-0.454900776 \backslash \mathrm{H}, 0.1635366202,2$. $1695014161,1.2934837982 \backslash \mathrm{C},-2.195803669,-1.5120723941,0.2976480646 \backslash \mathrm{H},-3$ $.1498308465,-1.4919348435,0.8319987323 \backslash \mathrm{H},-2.2853952577,-2.1998370733$, $0.5498503922 \backslash \mathrm{H},-1.4414060137,-1.9202486785,0.9919974323 \backslash \mathrm{H}, 0.2282741165$ $,-0.8293300748,1.1886316218 \backslash \backslash$ Version=DEC-AXP-OSF /1-G03RevB .03 $\backslash$ State $=1-$ $\mathrm{A} \backslash \mathrm{HF}=-899.7593089 \backslash \mathrm{RMSD}=5.209 \mathrm{e}-09 \backslash \mathrm{RMSF}=1.052 \mathrm{e}-05 \backslash \mathrm{Dipole}=1.660995,-0.189$ $7782,0.1348838 \backslash \mathrm{PG}=\mathrm{C} 01 \quad[\mathrm{X}(\mathrm{C} 4 \mathrm{H} 13 \mathrm{P} 1 \mathrm{~S} 1)] \backslash \backslash @$

\section{Equatorial $\cdot \mathrm{P}\left(\mathrm{CH}_{3}\right)_{3} \mathrm{SCH}_{3}(3)$}

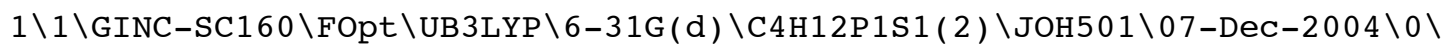
\\# B3LYP/6-31G* OPT FREQ MAXDISK=134217728\\pme3sch3_eq1 opt freq b31y $\mathrm{p} \backslash \backslash 0,2 \backslash \mathrm{C},-2.0984227793,0.4434847474,-0.6012454514 \backslash \mathrm{P},-0.082847253,0.424$ $1692139,-0.5861215799 \backslash \mathrm{C}, 1.8286518869,1.0510925461,-0.7141559405 \backslash \mathrm{S}, 0.17$ $81132049,-0.958991493,1.0488832904 \backslash \mathrm{C}, 0.0557834924,-0.739964441,-2.0330$ $940577 \backslash \mathrm{H},-0.7285259159,-1.5004856786,-2.0041076027 \backslash \mathrm{H}, 1.0335334268,-1.2$ $277278018,-2.0546607526 \backslash \mathrm{H},-0.0605761182,-0.1593097077,-2.9557803704 \backslash \mathrm{H}$, $-2.5084054338,-0.5721252767,-0.6179204244 \backslash \mathrm{H},-2.4400062151,0.9744511024$ $, 0.2914032374 \backslash \mathrm{H},-2.4125293731,1.0008586894,-1.4906736155 \backslash \mathrm{H}, 2.524609527$ $5,0.2066001458,-0.7621546857 \backslash \mathrm{H}, 1.9093673018,1.6690738872,-1.6152651027$ $\backslash \mathrm{H}, 2.043444017,1.6686789059,0.1621332168 \backslash \mathrm{C}, 0.0429245046,0.1956296548,2$ $.4715193414 \backslash \mathrm{H}, 0.1630220166,-0.411602475,3.3726898752 \backslash \mathrm{H}, 0.8316894228,0$. $9515792619,2.4466730567 \backslash \mathrm{H},-0.9363477669,0.6798795817,2.4992108695 \backslash \backslash$ Ver sion=DEC-AXP-OSF / 1-G03RevB.03 \State=2-A \HF=-899.1307301 \S2=0.756447\S2 $-1=0 . \backslash \mathrm{S} 2 \mathrm{~A}=0.750029 \backslash \mathrm{RMSD}=5.258 \mathrm{e}-09 \backslash \mathrm{RMSF}=2.435 \mathrm{e}-06 \backslash \mathrm{Dipole}=-0.0519553,0.3$ $189419,-0.1179674 \backslash \mathrm{PG}=\mathrm{C} 01 \quad[\mathrm{X}(\mathrm{C} 4 \mathrm{H} 12 \mathrm{P} 1 \mathrm{~S} 1)] \backslash \backslash @$

\section{Axial $\cdot \mathrm{P}\left(\mathrm{CH}_{3}\right)_{3} \mathrm{OCH}_{3}(4)$}

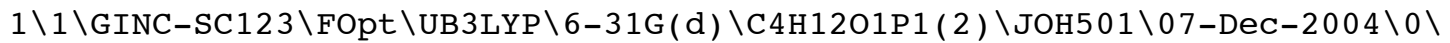
\\# B3LYP/6-31G* OPT FREQ MAXDISK=134217728\\pme3och3_ax1 opt freq b31y $\mathrm{p} \backslash \backslash 0,2 \backslash 0,-1.4790169652,0.1632564966,0.3691133117 \backslash \mathrm{P}, 0.315487307,0.07786$ $68465,0.081519281 \backslash \mathrm{C}, 2.1774527206,0.6126556118,0.0150272461 \backslash \mathrm{C}, 0.4803563$ $153,-1.2103593409,1.3914863641 \backslash \mathrm{C}, 0.2692499795,-0.8603851138,-1.5134502$ $511 \backslash \mathrm{H},-0.6406094902,-1.4643348818,-1.5547985793 \backslash \mathrm{H}, 1.1439420441,-1.5126$ $990882,-1.6129193267 \backslash \mathrm{H}, 0.2651606384,-0.1660248721,-2.3603385246 \backslash \mathrm{H},-0.4$ $354611625,-1.802657926,1.4402279716 \backslash \mathrm{H}, 0.6333357417,-0.7292264938,2.362$ $9753064 \backslash \mathrm{H}, 1.335660687,-1.8637895998,1.1905393 \backslash \mathrm{H}, 2.8528023825,-0.245350$ $9802,-0.1176101674 \backslash \mathrm{H}, 2.3181106022,1.3113042852,-0.8162171446 \backslash \mathrm{H}, 2.43484$ $3925,1.1291270304,0.9454954085 \backslash \mathrm{C},-2.1914756683,1.1972939226,-0.2780302$ $241 \backslash \mathrm{H},-3.231896493,1.1444143813,0.0632932282 \backslash \mathrm{H},-1.8004031935,2.1955078$ $771,-0.0307582607 \backslash \mathrm{H},-2.1891596477,1.0944451181,-1.3757837287 \backslash \backslash$ Version= DEC-AXP-OSF / 1-G03RevB.03 SState $=2-\mathrm{A} \backslash \mathrm{HF}=-576.1719763 \backslash \mathrm{S} 2=0.754072 \backslash \mathrm{S} 2-1=0$. $\backslash \mathrm{S} 2 \mathrm{~A}=0.750012 \backslash \mathrm{RMSD}=8.106 \mathrm{e}-09 \backslash \mathrm{RMSF}=6.208 \mathrm{e}-06 \backslash \mathrm{Dipole}=0.436136,-0.1178623$ ,$-0.3171714 \backslash \mathrm{PG}=\mathrm{C} 01[\mathrm{X}(\mathrm{C} 4 \mathrm{H} 1201 \mathrm{P} 1)] \backslash \backslash @$

\section{Axial $\mathrm{HP}\left(\mathrm{CH}_{3}\right)_{3} \mathrm{OCH}_{3}$}

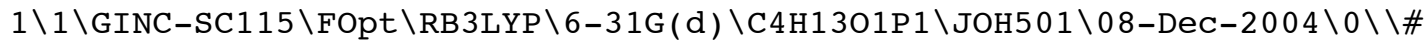
B3LYP/6-31G* OPT FREQ MAXDISK=134217728\\a_pme3och3_ax1 opt freq b3lyp $\backslash \backslash 0,1 \backslash 0,-1.478167231,-0.0292018112,0.0661844627 \backslash \mathrm{P}, 0.3111678875,0.14535$ $11566,0.0987141187 \backslash \mathrm{C}, 2.1686295962,0.6005408328,0.2104552323 \backslash \mathrm{C}, 0.233677$ $7908,-0.5116426367,1.8257639142 \backslash \mathrm{C}, 0.522341594,-1.1122862772,-1.2390683$ 
$281 \backslash \mathrm{H},-0.1997725216,-1.9166087348,-1.084546974 \backslash \mathrm{H}, 1.54013658,-1.5075841$ $83,-1.2801052037 \backslash \mathrm{H}, 0.2941701187,-0.6398598048,-2.2004907203 \backslash \mathrm{H},-0.64589$ $83234,-1.1415634443,1.953249588 \backslash \mathrm{H}, 0.155585244,0.3396214561,2.510665450$ $3 \backslash \mathrm{H}, 1.1507455388,-1.057836059,2.0622359057 \backslash \mathrm{H}, 2.8167547361,-0.249014108$ $8,0.4611303663 \backslash \mathrm{H}, 2.5038837208,1.0138373672,-0.7504805291 \backslash \mathrm{H}, 2.320661227$ $7,1.3788735705,0.970616112 \backslash \mathrm{C},-2.1943718573,0.6523048349,-0.9220859795 \backslash$ $\mathrm{H},-3.2216209431,0.8208122925,-0.5740710758 \backslash \mathrm{H},-1.7577765344,1.646075098$ $,-1.150231059 \backslash \mathrm{H},-2.2481420355,0.0954007718,-1.874500298 \backslash \mathrm{H}, 0.0674299856$ $, 1.4976923967,-0.3040480767 \backslash \backslash$ Version=DEC-AXP-OSF /1-G03RevB.03\State=1$\mathrm{A} \backslash \mathrm{HF}=-576.7885361 \backslash \mathrm{RMSD}=9.517 \mathrm{e}-09 \backslash \mathrm{RMSF}=1.004 \mathrm{e}-05 \backslash \mathrm{Dipole}=0.4750107,-0.07$ $48703,-0.1556153 \backslash \mathrm{PG}=\mathrm{C} 01[\mathrm{X}(\mathrm{C} 4 \mathrm{H} 1301 \mathrm{P} 1)] \backslash \backslash @$

\section{Equatorial $\cdot \mathrm{P}\left(\mathrm{CH}_{3}\right)_{3} \mathrm{OCH}_{3}(5)$}

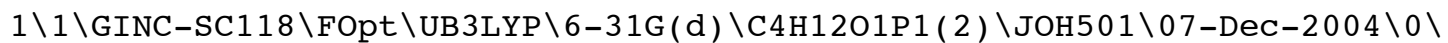
\\# B3LYP/6-31G* OPT FREQ MAXDISK=134217728\\pme3och3_eq1 opt freq b3ly $\mathrm{p} \backslash \backslash 0,2 \backslash \mathrm{C},-2.013994459,0.323852142,-0.3387680513 \backslash \mathrm{P},-0.044284007,0.30821$ $83098,-0.3991282131 \backslash \mathrm{C}, 1.8574994228,0.8505487813,-0.3134473696 \backslash 0,0.0792$ $583008,-0.7201946288,0.9355107374 \backslash \mathrm{C}, 0.0970379982,-0.9087793827,-1.7795$ $190617 \backslash \mathrm{H},-0.2097049057,-1.8927583713,-1.4078854316 \backslash \mathrm{H}, 1.1195356296,-0.9$ $767461056,-2.1585286122 \backslash \mathrm{H},-0.5722277331,-0.6250277995,-2.597793816 \backslash \mathrm{H},-$ $2.4101869459,-0.6992005499,-0.3197754099 \backslash \mathrm{H},-2.344647919,0.8584087054,0$ $.5569019111 \backslash \mathrm{H},-2.384357486,0.8586108508,-1.2195816633 \backslash \mathrm{H}, 2.5057683548,-$ $0.0255086139,-0.1890936919 \backslash \mathrm{H}, 2.095170787,1.3714383439,-1.2469314233 \backslash \mathrm{H}$, $2.0094916835,1.544265053,0.5186483723 \backslash \mathrm{C}, 0.0651422035,-0.1419241808,2.2$ $313876715 \backslash \mathrm{H}, 0.1223746556,-0.9644941855,2.9503787582 \backslash \mathrm{H}, 0.9235757851,0.5$ $242242594,2.3961082635 \backslash \mathrm{H},-0.8587091993,0.4228866382,2.4224709075 \backslash \backslash$ Vers ion $=\mathrm{DEC}-\mathrm{AXP}-\mathrm{OSF} / 1-\mathrm{G} 03 \mathrm{RevB} .03 \backslash \mathrm{State}=2-\mathrm{A} \backslash \mathrm{HF}=-576.1564681 \backslash \mathrm{S} 2=0.754307 \backslash \mathrm{S} 2-$ $1=0 . \backslash \mathrm{S} 2 \mathrm{~A}=0.750014 \backslash \mathrm{RMSD}=6.400 \mathrm{e}-09 \backslash \mathrm{RMSF}=4.579 \mathrm{e}-06 \backslash \mathrm{Dipole}=-0.0036615,0.10$ $84439,0.0220184 \backslash \mathrm{PG}=\mathrm{C} 01 \quad[\mathrm{X}(\mathrm{C} 4 \mathrm{H} 1201 \mathrm{P} 1)] \backslash \backslash @$

\section{Axial $\cdot \mathrm{P}\left(\mathrm{CH}_{3}\right)_{3} \mathrm{CN}(6)$}

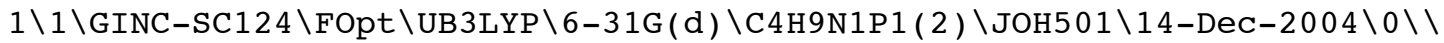
\# B3LYP/6-31G* OPT FREQ MAXDISK=134217728\\pme3cn_ax4 opt freq b3lyp \\ $0,2 \backslash P, 0.2644952,-0.0398985156,0 . \backslash \mathrm{C},-1.4557154782,-0.9319553873,0 . \backslash \mathrm{N},-2$ $.1565906551,-1.874554635,0 . \backslash \mathrm{C}, 2.1972803043,-0.0398419698,0 . \backslash \mathrm{H}, 2.600664$ $8399,0.9817685648,0 . \backslash \mathrm{H}, 2.5501013869,-0.571326129,-0.8885548257 \backslash \mathrm{H}, 2.550$ $1013869,-0.571326129,0.8885548257 \backslash \mathrm{C},-0.037445992,1.0254102895,-1.47542$ $72798 \backslash \mathrm{C},-0.037445992,1.0254102895,1.4754272798 \backslash \mathrm{H}, 0.6359663517,1.887897$ $9518,-1.4628041967 \backslash \mathrm{H}, 0.6359663517,1.8878979518,1.4628041967 \backslash \mathrm{H}, 0.152057$ $737,0.447723939,-2.3859040722 \backslash \mathrm{H}, 0.152057737,0.447723939,2.3859040722 \backslash \mathrm{H}$ $,-1.0741231289,1.3679303799,-1.492583675 \backslash \mathrm{H},-1.0741231289,1.3679303799$, $1.492583675 \backslash \backslash$ Version=DEC-AXP-OSF $/ 1-G 03$ RevB.03 $\backslash$ State $=2-A^{\prime} \backslash H F=-553.87066$ $77 \backslash S 2=0.755578 \backslash S 2-1=0 . \backslash S 2 A=0.750022 \backslash R M S D=4.764 e-09 \backslash R M S F=4.693 e-06 \backslash D i p o$ $l e=1.597852,1.601244,0 . \backslash \mathrm{PG}=\mathrm{CS} \quad[\mathrm{SG}(\mathrm{C} 2 \mathrm{H} 1 \mathrm{~N} 1 \mathrm{P} 1), \mathrm{X}(\mathrm{C} 2 \mathrm{H} 8)] \backslash \backslash \mathrm{a}$

\section{Axial $\mathrm{HP}\left(\mathrm{CH}_{3}\right)_{3} \mathrm{CN}$}

$1 \backslash 1 \backslash G I N C-S C 96 \backslash F O p t \backslash R B 3 L Y P \backslash 6-31 G(d) \backslash C 4 H 10 N 1 P 1 \backslash J O H 501 \backslash 15-D e C-2004 \backslash 0 \backslash \backslash \# B$ 3LYP/6-31G* OPT FREQ MAXDISK $=65536000 \backslash \backslash \mathrm{a} \_$pme3cn_ax4 opt freq b3lyp $\backslash \backslash 0$, $1 \backslash \mathrm{P},-0.3100002883,-0.2990125239,0 . \backslash \mathrm{H}, 0.08928805 \overline{1} 8,-1.6497877747,0 . \backslash \mathrm{C}, 1$ $.745348909,0.0292818651,0 . \backslash \mathrm{N}, 2.9071431805,0.1431007357,0 . \backslash \mathrm{C},-2.1503655$ $47,-0.7813311869,0 . \backslash \mathrm{H},-2.8170133995,0.0898080691,0 . \backslash \mathrm{C},-0.3281100265,0$. $6248605464,1.5975127132 \backslash \mathrm{C},-0.3281100265,0.6248605464,-1.5975127132 \backslash \mathrm{H},-$ $2.3814651327,-1.3867024728,0.885832184 \backslash \mathrm{H},-2.3814651327,-1.3867024728,-$ $0.885832184 \backslash \mathrm{H}, 0.2614707884,0.063451663,2.3274644785 \backslash \mathrm{H}, 0.2614707884,0.0$ $63451663,-2.3274644785 \backslash \mathrm{H}, 0.1646065144,1.5893928186,1.4529905906 \backslash \mathrm{H}, 0.16$ $46065144,1.5893928186,-1.4529905906 \backslash \mathrm{H},-1.3470383928,0.7625738852,1.966$ $9566663 \backslash \mathrm{H},-1.3470383928,0.7625738852,-1.9669566663 \backslash \backslash$ Version=DEC-AXP-OS 
F / 1-G03RevB.03 \State $=1-A^{\prime} \backslash H F=-554.4812662 \backslash$ RMSD $=1.820 e-09 \backslash R M S F=8.541 e-0$ $5 \backslash$ Dipole $=-2.2080835,0.0399664,0 . \backslash \mathrm{PG}=\mathrm{CS} \quad[\mathrm{SG}(\mathrm{C} 2 \mathrm{H} 2 \mathrm{~N} 1 \mathrm{P} 1), \mathrm{X}(\mathrm{C} 2 \mathrm{H} 8)] \backslash \backslash @$

\section{Equatorial $\cdot \mathrm{P}\left(\mathrm{CH}_{3}\right)_{3} \mathrm{CN}(7)$}

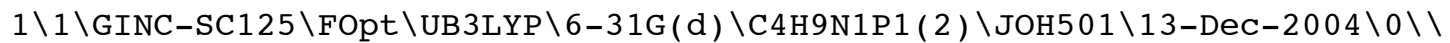
\# B3LYP/6-31G* OPT FREQ MAXDISK=65536000 \\me3cn_eq3 opt freq b3lyp $\backslash \backslash 0$ , $2 \backslash \mathrm{P}, 0.4588801129,0.2334530387,0 . \backslash \mathrm{C}, 0.9247454862,-1.5691348081,0 . \backslash \mathrm{C},-1$ $.3458850865,0.2107238079,0 . \backslash \mathrm{N},-2.5099137435,0.2234109417,0 . \backslash \mathrm{H}, 2.018254$ $7823,-1.6398339748,0 . \backslash \mathrm{C}, 0.5516231829,0.4746836129,-1.9778064108 \backslash \mathrm{C}, 0.55$ $16231829,0.4746836129,1.9778064108 \backslash \mathrm{H}, 0.5442849764,-2.0727597989,-0.891$ $4780334 \backslash \mathrm{H}, 0.5442849764,-2.0727597989,0.8914780334 \backslash \mathrm{H}, 1.6003813332,0.359$ $9648433,-2.2687710457 \backslash \mathrm{H}, 1.6003813332,0.3599648433,2.2687710457 \backslash \mathrm{H},-0.07$ $40966628,-0.260777162,-2.4963056707 \backslash \mathrm{H},-0.0740966628,-0.260777162,2.496$ $3056707 \backslash \mathrm{H}, 0.2170799205,1.4877843417,-2.2136261809 \backslash \mathrm{H}, 0.2170799205,1.487$ $7843417,2.2136261809 \backslash \backslash$ Version=DEC-AXP-OSF/1-G03RevB.03 $\backslash$ State=2-A ' $\backslash \mathrm{HF}=-$ $553.860441 \backslash \mathrm{S} 2=0.755441 \backslash \mathrm{S} 2-1=0 . \backslash \mathrm{S} 2 \mathrm{~A}=0.750021 \backslash \mathrm{RMSD}=8.421 \mathrm{e}-09 \backslash \mathrm{RMSF}=1.084 \mathrm{e}$ $-05 \backslash$ Dipole $=1.4443331,-0.4067133,0 . \backslash P G=C S \quad[S G(C 2 H 1 N 1 P 1), X(C 2 H 8)] \backslash \backslash @$

\section{Axial $\cdot \mathrm{P}\left(\mathrm{CH}_{3}\right)_{3}\left(\mathrm{C}_{6} \mathrm{H}_{5}\right)(8)$}

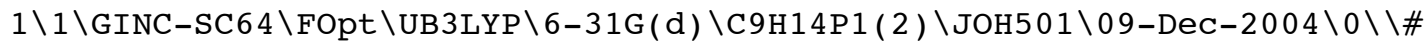
B3LYP/6-31G* OPT FREQ MAXDISK=134217728\\pme3ph_ax3 opt freq b3lyp \\0, $2 \backslash \mathrm{P}, 1.2770953134,-0.5676180502,0 . \backslash \mathrm{C}, 3.261713504 \overline{7},-0.5690425901,0 . \backslash \mathrm{C},-0$ $.4755940703,0.2449809771,0 . \backslash \mathrm{C},-2.7882022201,1.8737849847,0 . \backslash \mathrm{H}, 3.682094$ $0589,-1.5849928333,0 . \backslash \mathrm{H},-3.6797140813,2.4950301425,0 . \backslash \mathrm{C}, 1.0840042827,-$ $1.6915463949,1.46517182 \backslash \mathrm{C}, 1.0840042827,-1.6915463949,-1.46517182 \backslash \mathrm{C},-1$. $0580664238,0.6811327982,1.2056570629 \backslash \mathrm{C},-1.0580664238,0.6811327982,-1.2$ $056570629 \backslash \mathrm{C},-2.2050497858,1.4739296526,1.2056263854 \backslash \mathrm{C},-2.2050497858,1$. $4739296526,-1.2056263854 \backslash \mathrm{H}, 3.6043133768,-0.031006467,0.8892619019 \backslash \mathrm{H}, 3$. $6043133768,-0.031006467,-0.8892619019 \backslash \mathrm{H}, 1.7384897318,-2.5608545653,1.3$ $476795347 \backslash \mathrm{H}, 1.7384897318,-2.5608545653,-1.3476795347 \backslash \mathrm{H}, 1.3768193104,-1$ $.1743543334,2.3847754202 \backslash \mathrm{H}, 1.3768193104,-1.1743543334,-2.3847754202 \backslash \mathrm{H}$, $0.0506446731,-2.0316458051,1.5678874803 \backslash \mathrm{H}, 0.0506446731,-2.0316458051$, $1.5678874803 \backslash \mathrm{H},-0.6199414429,0.3911974356,2.1587253428 \backslash \mathrm{H},-0.6199414429$ $, 0.3911974356,-2.1587253428 \backslash \mathrm{H},-2.6488105703,1.7785140066,2.1510918561 \backslash$ $\mathrm{H},-2.6488105703,1.7785140066,-2.1510918561 \backslash \backslash$ Version=DEC-AXP-OSF / 1-G03R evB.03 $\backslash$ state $=2-A^{\prime} \backslash H F=-692.6708459 \backslash S 2=0.756262 \backslash S 2-1=0 . \backslash S 2 A=0.750034 \backslash R M S$ $\mathrm{D}=4.483 \mathrm{e}-09 \backslash \mathrm{RMSF}=6.082 \mathrm{e}-06 \backslash \mathrm{Dipole}=0.2789852,-0.553024,0 . \backslash \mathrm{PG}=\mathrm{CS} \quad[\mathrm{SG}(\mathrm{C} 3 \mathrm{H}$ $2 \mathrm{P} 1), \mathrm{X}(\mathrm{C} 6 \mathrm{H} 12)] \backslash \backslash @$

\section{Axial $\mathrm{HP}\left(\mathrm{CH}_{3}\right)_{3}\left(\mathrm{C}_{6} \mathrm{H}_{5}\right)$}

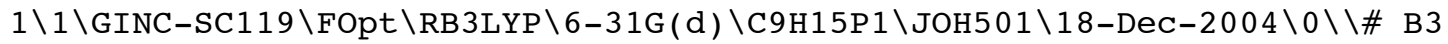
LYP/6-31G* OPT FREQ MAXDISK=134217728\\a_pme3ph_ax34 opt freq b3lyp $\backslash \backslash 0$ $, 1 \backslash \mathrm{P}, 1.453395327,0.1208558366,0 . \backslash \mathrm{H}, 1.311156575, \overline{1} .5560831798,0 . \backslash \mathrm{C}, 3.366$ $8839358,0.4568299546,0 . \backslash \mathrm{H}, 3.9918626588,-0.4459618782,0 . \backslash \mathrm{C},-0.525774879$ $1,0.1215008419,0 . \backslash \mathrm{C},-3.3520016284,0.2895880877,0 . \backslash \mathrm{H},-4.4372345662,0.35$ $33131329,0 . \backslash \mathrm{C},-1.3267285554,-1.033432658,0 . \backslash \mathrm{C},-2.7202703453,-0.9574714$ $968,0 . \backslash \mathrm{C},-2.5784470001,1.4495705525,0 . \backslash \mathrm{C},-1.1827344108,1.3549213444,0$. $\backslash \mathrm{H},-0.8624159259,-2.0203682471,0 . \backslash \mathrm{H},-3.3154403231,-1.8681267466,0 . \backslash \mathrm{H},-$ $3.0595567177,2.4252438339,0 . \backslash \mathrm{H},-0.5913696234,2.2728416037,0 . \backslash \mathrm{C}, 1.53924$ $61922,-0.8491265934,1.5889061318 \backslash \mathrm{C}, 1.5392461922,-0.8491265934,-1.58890$ $61318 \backslash \mathrm{H}, 3.6275091407,1.0543149934,-0.8847231979 \backslash \mathrm{H}, 3.6275091407,1.05431$ $49934,0.8847231979 \backslash \mathrm{H}, 2.4858580919,-1.3905045357,-1.6621456702 \backslash \mathrm{H}, 2.4858$ $580919,-1.3905045357,1.6621456702 \backslash \mathrm{H}, 1.4942073885,-0.1252272179,-2.4105$ $272735 \backslash \mathrm{H}, 1.4942073885,-0.1252272179,2.4105272735 \backslash \mathrm{H}, 0.6952008845,-1.531$ $274772,-1.6973912189 \backslash \mathrm{H}, 0.6952008845,-1.531274772,1.6973912189 \backslash \backslash$ Version =DEC-AXP-OSF / 1-G03RevB .03 \State=1-A ' $\backslash \mathrm{HF}=-693.2740643 \backslash \mathrm{RMSD}=7.962 e-09 \backslash \mathrm{RM}$ $\mathrm{SF}=3.013 e-05 \backslash \mathrm{Dipole}=0.2044245,-0.3138971,0 . \backslash \mathrm{PG}=\mathrm{CS} \quad[\mathrm{SG}(\mathrm{C} 7 \mathrm{H} 7 \mathrm{P} 1), \mathrm{X}(\mathrm{C} 2 \mathrm{H} 8)]$ 
$\backslash \backslash @$

\section{Equatorial $\cdot \mathrm{P}\left(\mathrm{CH}_{3}\right)_{3}\left(\mathrm{C}_{6} \mathrm{H}_{5}\right)(9)$}

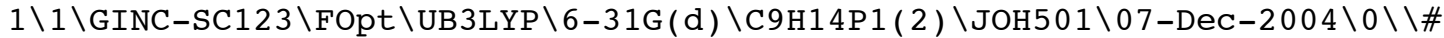
B3LYP/6-31G* OPT FREQ MAXDISK=134217728\\pme3ph_eq1 opt freq b3lyp $\backslash \backslash 0$ , $2 \backslash \mathrm{C},-2.1418376712,0.518651679,-1.490983869 \backslash \mathrm{P},-0.2357841545,1.03263052$ $14,-1.1187502811 \backslash \mathrm{C}, 1.4363293686,2.0911377312,-0.7965979784 \backslash \mathrm{C},-0.019410$ $825,-0.107663968,0.321549595 \backslash \mathrm{C}, 0.4215768861,0.1173419798,-2.6008877526$ $\backslash \mathrm{H},-0.010997928,-0.885393268,-2.6668502652 \backslash \mathrm{H}, 1.5121319423,0.0401645658$ $,-2.5858343082 \backslash \mathrm{H}, 0.1344317073,0.6696895626,-3.5027443974 \backslash \mathrm{H},-2.25296751$ $6,-0.5694825409,-1.5763052208 \backslash \mathrm{H},-2.7757326863,0.893953409,-0.683449194$ $8 \backslash \mathrm{H},-2.4364835136,1.0023344522,-2.4278302745 \backslash \mathrm{H}, 2.3065912125,1.43729527$ $56,-0.6596308219 \backslash \mathrm{H}, 1.5930043828,2.7511494134,-1.6561652583 \backslash \mathrm{H}, 1.2850347$ $77,2.6979621186,0.1003584917 \backslash \mathrm{C},-0.8042683436,0.0862116848,1.4689171014$ $\backslash \mathrm{C},-0.6505367642,-0.7298817903,2.5904121408 \backslash \mathrm{C}, 0.2901485364,-1.76122833$ $45,2.5828147514 \backslash \mathrm{C}, 0.9263957693,-1.1433178476,0.3309529177 \backslash \mathrm{C}, 1.07763651$ $79,-1.9658694657,1.4492278242 \backslash \mathrm{H},-1.5432449174,0.883616692,1.4894764062$ $\backslash \mathrm{H},-1.2670462952,-0.5598376684,3.4694322796 \backslash \mathrm{H}, 0.4090766295,-2.39981360$ $57,3.4541438305 \backslash \mathrm{H}, 1.5538740746,-1.3168364781,-0.5392108113 \backslash \mathrm{H}, 1.8128896$ $022,-2.7665497606,1.4334353774 \backslash \backslash$ Version=DEC-AXP-OSF/1-G03RevB.03\State $=2-\mathrm{A} \backslash \mathrm{HF}=-692.6671483 \backslash \mathrm{S} 2=0.755335 \backslash \mathrm{S} 2-1=0 . \backslash \mathrm{S} 2 \mathrm{~A}=0.75002 \backslash \mathrm{RMSD}=4.615 \mathrm{e}-09 \backslash \mathrm{RM}$ $\mathrm{SF}=4.567 e-05 \backslash \mathrm{Dipole}=0.1419923,-0.3356845,-0.0348715 \backslash \mathrm{PG}=\mathrm{C} 01 \quad[\mathrm{X}(\mathrm{C} 9 \mathrm{H} 14 \mathrm{P} 1)$ ]$\backslash \backslash @$

\section{Axial $\cdot \mathrm{P}\left(\mathrm{CH}_{3}\right)_{3} \mathrm{~F}(10)$}

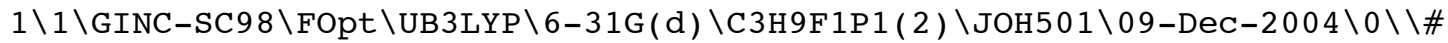
B3LYP/6-31G* OPT FREQ MAXDISK=65536000\\pme3f_ax2 opt freq b3lyp \\0,2 $\backslash P,-0.0049192218,0 .,-0.2917887575 \backslash \mathrm{C},-0.1697451847,-1.468680657,0.80642$ $46919 \backslash \mathrm{C},-0.1697451847,1.468680657,0.8064246919 \backslash \mathrm{F},-1.6653947816,0 .,-0.7$ $745875179 \backslash \mathrm{C}, 1.9150370221,0 .,-0.4789522923 \backslash \mathrm{H}, 2.429418192,0 ., 0.493615696$ $1 \backslash \mathrm{H}, 2.2197354017,0.8867786828,-1.0441817187 \backslash \mathrm{H}, 2.2197354017,-0.88677868$ $28,-1.0441817187 \backslash \mathrm{H}, 0.6111354348,1.4846875771,1.5739535346 \backslash \mathrm{H}, 0.61113543$ $48,-1.4846875771,1.5739535346 \backslash \mathrm{H},-0.0882244892,2.3848513085,0.212191476$ $\backslash \mathrm{H},-0.0882244892,-2.3848513085,0.212191476 \backslash \mathrm{H},-1.1528247205,1.460233448$ $9,1.2835970972 \backslash \mathrm{H},-1.1528247205,-1.4602334489,1.2835970972 \backslash \backslash$ Version=DEC -AXP-OSF / 1-G03RevB.03\State $=2-A^{\prime} \backslash H F=-560.9029477 \backslash S 2=0.753048 \backslash S 2-1=0 . \backslash S$ $2 \mathrm{~A}=0.750007 \backslash \mathrm{RMSD}=5.682 \mathrm{e}-09 \backslash \mathrm{RMSF}=1.817 \mathrm{e}-05 \backslash \mathrm{Dipole}=0.7599122,0 ., 0.655670$ $4 \backslash P G=C S \quad[S G(C 1 H 1 F 1 P 1), X(C 2 H 8)] \backslash \backslash @$

\section{Axial $\mathrm{HP}\left(\mathrm{CH}_{3}\right)_{3} \mathrm{~F}$}

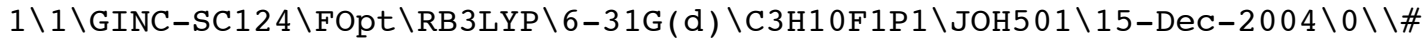
B3LYP/6-31G* OPT FREQ MAXDISK=65536000\\a_pme3f_ax22 opt freq b3lyp $\backslash \backslash 0$ $, 1 \backslash \mathrm{P},-0.2298015934,-0.1687846644,0 . \backslash \mathrm{H},-1 . \overline{4} 06466 \overline{9} 721,-0.9545814194,0 . \backslash \mathrm{C}$ $, 0.4492015608,0.4896506946,1.5844465488 \backslash \mathrm{C}, 0.4492015608,0.4896506946,-1$ $.5844465488 \backslash \mathrm{F},-1.313542482,1.1820709845,0 . \backslash \mathrm{C}, 0.8129560097,-1.762777093$ $4,0 . \backslash \mathrm{H}, 1.8924480054,-1.5635212865,0 . \backslash \mathrm{H}, 0.5812317202,-2.3704700537,-0.8$ $851532745 \backslash \mathrm{H}, 0.5812317202,-2.3704700537,0.8851532745 \backslash \mathrm{H}, 1.3273757719,-0$. $0583722811,-1.9314810872 \backslash \mathrm{H}, 1.3273757719,-0.0583722811,1.9314810872 \backslash \mathrm{H},-$ $0.3380215077,0.4377621058,-2.3423784614 \backslash \mathrm{H},-0.3380215077,0.4377621058,2$ $.3423784614 \backslash \mathrm{H}, 0.6867992245,1.5471242475,-1.4468395094 \backslash \mathrm{H}, 0.6867992245,1$ $.5471242475,1.4468395094 \backslash \backslash$ Version=DEC-AXP-OSF/1-G03RevB.03\State=1-A ' $\mathrm{HF}=-561.5263309 \backslash \mathrm{RMSD}=2.096 \mathrm{e}-09 \backslash \mathrm{RMSF}=1.259 \mathrm{e}-05 \backslash \mathrm{Dipole}=0.665767,-0.38846$ $77,0 . \backslash \mathrm{PG}=\mathrm{CS} \quad[\mathrm{SG}(\mathrm{C} 1 \mathrm{H} 2 \mathrm{~F} 1 \mathrm{P} 1), \mathrm{X}(\mathrm{C} 2 \mathrm{H} 8)] \backslash \backslash \mathrm{a}$

\section{Equatorial $\cdot \mathrm{P}\left(\mathrm{CH}_{3}\right)_{3} \mathrm{~F}(11)$}

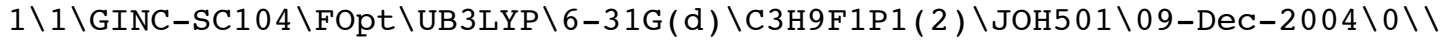


\# B3LYP/6-31G* OPT FREQ MAXDISK=65536000\\pme3f_eq2 opt freq b3lyp \\0, $2 \backslash P, 0 .,-0.0295689069,-0.2936303655 \backslash F, 0 \ldots,-1.0073 \overline{2} 6657,1.0441368333 \backslash \mathrm{C}, 0$. $, 1.6901931773,0.3597926719 \backslash \mathrm{H}, 0 ., 1.6705741401,1.4570706761 \backslash \mathrm{H}, 0.89190927$ $05,2.2277395364,0.0252499865 \backslash \mathrm{H},-0.8919092705,2.2277395364,0.0252499865$ $\backslash \mathrm{C}, 1.8918757386,-0.3522515706,-0.4669768087 \backslash \mathrm{C},-1.8918757386,-0.3522515$ $706,-0.4669768087 \backslash \mathrm{H}, 2.4153062772,-0.144094703,0.4746112405 \backslash \mathrm{H},-2.415306$ $2772,-0.144094703,0.4746112405 \backslash \mathrm{H}, 2.2841359441,0.2805351823,-1.26913686$ $33 \backslash \mathrm{H},-2.2841359441,0.2805351823,-1.2691368633 \backslash \mathrm{H}, 2.046333872,-1.4018004$ $357,-0.7331648735 \backslash \mathrm{H},-2.046333872,-1.4018004357,-0.7331648735 \backslash \backslash$ Version= DEC-AXP-OSF / 1-G03RevB.03 \State $=2-\mathrm{A}^{\prime} \backslash \mathrm{HF}=-560.8924869 \backslash \mathrm{S} 2=0.75288 \backslash \mathrm{S} 2-1=0$. $\backslash \mathrm{S} 2 \mathrm{~A}=0.750006 \backslash \mathrm{RMSD}=8.868 \mathrm{e}-09 \backslash \mathrm{RMSF}=4.248 \mathrm{e}-05 \backslash \mathrm{Dipole}=0,0.5424467,-0.251$ $6663 \backslash \mathrm{PG}=\mathrm{CS} \quad[\mathrm{SG}(\mathrm{C} 1 \mathrm{H} 1 \mathrm{~F} 1 \mathrm{P} 1), \mathrm{X}(\mathrm{C} 2 \mathrm{H} 8)] \backslash \backslash \mathrm{Q}$

\section{Axial $\cdot \mathrm{P}\left(\mathrm{CH}_{3}\right)_{3} \mathrm{OH}(12)$}

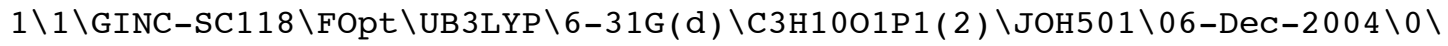
\\# B3LYP/6-31G* OPT FREQ MAXDISK=134217728\\pme3oh_ax1 opt freq b3lyp \ $\backslash 0,2 \backslash 0,-1.8738792216,0.2749593859,0.1570424797 \backslash \mathrm{P},-\overline{0} .0777063821,0.27473$ $73368,-0.0157434396 \backslash \mathrm{C}, 1.7356360294,0.9645193787,-0.0194210028 \backslash \mathrm{C}, 0.1040$ $927445,-0.8559797813,1.4294763538 \backslash \mathrm{C}, 0.0668767735,-0.83155147,-1.489954$ $5269 \backslash \mathrm{H},-0.7971429767,-1.4995383652,-1.5264340287 \backslash \mathrm{H}, 0.9869197923,-1.426$ $2401088,-1.4542186646 \backslash \mathrm{H}, 0.0819154901,-0.2340600219,-2.407941024 \backslash \mathrm{H},-0.7$ $480978867,-1.5385886579,1.4671288994 \backslash \mathrm{H}, 0.1146104505,-0.2710245193,2.35$ $48829805 \backslash \mathrm{H}, 1.0351579542,-1.4288643418,1.3691542809 \backslash \mathrm{H}, 2.4872474165,0.16$ $12972808,-0.0162915227 \backslash \mathrm{H}, 1.8784689954,1.5867343041,-0.9089606425 \backslash \mathrm{H}, 1.8$ $804053484,1.5917880107,0.8663001199 \backslash \mathrm{H},-2.202488364,1.0758325158,-0.284$ $4135862 \backslash \backslash$ Version=DEC-AXP-OSF $/ 1-G 03$ RevB .03 $\backslash$ State $=2-A \backslash H F=-536.8673677 \backslash \mathrm{S} 2$ $=0.754009 \backslash \mathrm{S} 2-1=0 . \backslash \mathrm{S} 2 \mathrm{~A}=0.750012 \backslash \mathrm{RMSD}=4.267 \mathrm{e}-09 \backslash \mathrm{RMSF}=5.697 \mathrm{e}-06 \backslash \mathrm{Dipole}=0$. $4733346,0.0429973,-0.2931307 \backslash \mathrm{PG}=\mathrm{C} 01[\mathrm{X}(\mathrm{C} 3 \mathrm{H} 1001 \mathrm{P} 1)] \backslash \backslash @$

\section{Axial $\mathrm{HP}\left(\mathrm{CH}_{3}\right)_{3} \mathrm{OH}$}

$1 \backslash 1 \backslash G I N C-S C 121 \backslash F O p t \backslash R B 3 L Y P \backslash 6-31 G(d) \backslash C 3 H 1101 P 1 \backslash J O H 501 \backslash 07-D e C-2004 \backslash 0 \backslash \backslash \#$ B3LYP/6-31G* OPT FREQ MAXDISK=134217728\\a_pme3oh_ax1 opt freq b3lyp \\ $0,1 \backslash 0,-1.8257879633,0.0503814346,-0.0354241267 \backslash \mathrm{P},-0.0605529381,0.25942$ $8975,-0.0602937143 \backslash \mathrm{C}, 1.7983034593,0.7417955287,-0.0069404443 \backslash \mathrm{C},-0.0594$ $722901,-0.4127268267,1.6638438705 \backslash \mathrm{C}, 0.1391871494,-0.9779267706,-1.4207$ $97519 \backslash \mathrm{H},-0.5371380799,-1.8145358832,-1.2349035397 \backslash \mathrm{H}, 1.1696900819,-1.32$ $70753073,-1.5252007464 \backslash \mathrm{H},-0.1675735204,-0.5106732194,-2.3633170151 \backslash \mathrm{H},-$ $0.8858562923,-1.1121421105,1.7923188217 \backslash \mathrm{H},-0.2109415305,0.4249020877,2$ $.3532350645 \backslash \mathrm{H}, 0.8994240777,-0.8828066122,1.8958814151 \backslash \mathrm{H}, 2.46426368,-0$. $1033715235,0.2107257278 \backslash \mathrm{H}, 2.0942693046,1.1710496996,-0.9740587494 \backslash \mathrm{H}, 1$. $9674650638,1.5123474348,0.7578612975 \backslash \mathrm{H},-2.2355292623,0.6234305222,-0.7$ $02479774 \backslash \mathrm{H},-0.3115856571,1.6175372223,-0.438899217 \backslash \backslash$ Version=DEC-AXP-OS $\mathrm{F} / 1-\mathrm{G} 03 \mathrm{RevB} .03 \backslash \mathrm{State}=1-\mathrm{A} \backslash \mathrm{HF}=-537.4795921 \backslash \mathrm{RMSD}=3.656 \mathrm{e}-09 \backslash \mathrm{RMSF}=9.222 \mathrm{e}-06$ $\backslash$ Dipole $=0.3631032,0.0887461,-0.3179666 \backslash \mathrm{PG}=\mathrm{C} 01[\mathrm{X}(\mathrm{C} 3 \mathrm{H} 1101 \mathrm{P} 1)] \backslash \backslash @$

\section{Equatorial $\cdot \mathrm{P}\left(\mathrm{CH}_{3}\right)_{3} \mathrm{OH}(13)$}

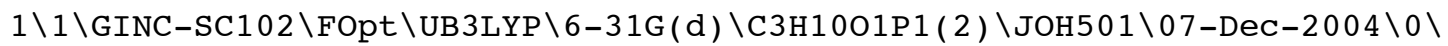
\\# B3LYP/6-31G* OPT FREQ MAXDISK=134217728\\pme3oh_eq1 opt freq b3lyp $\backslash 0,2 \backslash \mathrm{C},-1.9800628098,0.2986355509,-0.0340553366 \backslash \mathrm{P},-0.0438760031,0.2829$ $861347,-0.0425230751 \backslash \mathrm{C}, 1.8555701591,0.7876810655,0.1972078319 \backslash 0,-0.080$ $1771659,-0.5940987066,1.3956274507 \backslash \mathrm{C}, 0.1708948626,-0.9102805458,-1.443$ $8568055 \backslash \mathrm{H},-0.1724776462,-1.9035353229,-1.1313108015 \backslash \mathrm{H}, 1.2133321778,-0$. $983260243,-1.7657473916 \backslash \mathrm{H},-0.4411819848,-0.593637257,-2.2951833217 \backslash \mathrm{H},-$ $2.3785251363,-0.7200116095,0.0532957622 \backslash \mathrm{H},-2.321145369,0.8920241125,0$. $8182571452 \backslash \mathrm{H},-2.3297248603,0.7632155818,-0.9611995734 \backslash \mathrm{H}, 2.5167267491,-$ $0.0805416375,0.3441772086 \backslash \mathrm{H}, 2.1743306051,1.324167595,-0.7015600081 \backslash \mathrm{H}, 1$ $.9411088722,1.4621529784,1.0554553868 \backslash \mathrm{H}, 0.8187006949,-0.7087929888,1.7$ 
$408679765 \backslash \backslash$ Version=DEC-AXP-OSF $/ 1-G 03 R e v B .03 \backslash$ State $=2-A \backslash H F=-536.8574243 \backslash$ $\mathrm{S} 2=0.753522 \backslash \mathrm{S} 2-1=0 . \backslash \mathrm{S} 2 \mathrm{~A}=0.750009 \backslash \mathrm{RMSD}=7.919 \mathrm{e}-09 \backslash \mathrm{RMSF}=9.752 \mathrm{e}-06 \backslash \mathrm{Dipole}=$ $0.4888267,-0.1275575,-0.2545761 \backslash \mathrm{PG}=\mathrm{C} 01[\mathrm{X}(\mathrm{C} 3 \mathrm{H} 1001 \mathrm{P} 1)] \backslash \backslash @$

\section{Axial $\cdot \mathrm{P}\left(\mathrm{CH}_{3}\right)_{3} \mathrm{CF}_{3}(14)$}

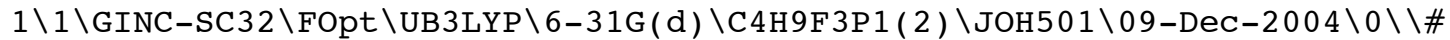
B3LYP/6-31G* OPT FREQ MAXDISK=134217728\\pme3cf3_ax3 opt freq b3lyp $\backslash$ $0,2 \backslash \mathrm{P},-0.8237587663,0 \ldots,-0.2207057149 \backslash \mathrm{C}, 1.2489136547,0,-0.1660188537 \backslash \mathrm{F}$ $, 1.8101104922,0.1 .0815200741 \backslash \mathrm{C},-2.6442992323,0 .,-0.8921953277 \backslash \mathrm{H},-3.37$ $82777989,0 .,-0.0752472481 \backslash \mathrm{F}, 1.7203688049,1.0918681164,-0.8054221945 \backslash \mathrm{F}$, $1.7203688049,-1.0918681164,-0.8054221945 \backslash \mathrm{C},-0.9657279227,1.4714235156$, $0.8997375999 \backslash \mathrm{C},-0.9657279227,-1.4714235156,0.8997375999 \backslash \mathrm{H},-0.112369916$ $2,1.5245519271,1.5796569954 \backslash \mathrm{H},-0.1123699162,-1.5245519271,1.5796569954$ $\backslash \mathrm{H},-0.9899687529,2.3869587285,0.3000132606 \backslash \mathrm{H},-0.9899687529,-2.38695872$ $85,0.3000132606 \backslash \mathrm{H},-1.8884760034,1.4164907729,1.4855067485 \backslash \mathrm{H},-1.8884760$ $034,-1.4164907729,1.4855067485 \backslash \mathrm{H},-2.790147871,0.8884217579,-1.51408415$ $75 \backslash \mathrm{H},-2.790147871,-0.8884217579,-1.5140841575 \backslash \backslash$ Version=DEC-AXP-OSF / 1-G 03RevB.03 \State $=2-A^{\prime} \backslash H F=-798.6570833 \backslash S 2=0.755547 \backslash S 2-1=0 . \backslash S 2 A=0.750019 \backslash$ $\mathrm{RMSD}=3.534 \mathrm{e}-09 \backslash \mathrm{RMSF}=2.390 \mathrm{e}-05 \backslash \mathrm{Dipole}=-1.1311491,0 ., 0.3878745 \backslash \mathrm{PG}=\mathrm{CS}$ [SG $(\mathrm{C} 2 \mathrm{H} 1 \mathrm{~F} 1 \mathrm{P} 1), \mathrm{X}(\mathrm{C} 2 \mathrm{H} 8 \mathrm{~F} 2)] \backslash \backslash @$

\section{Axial $\mathrm{HP}\left(\mathrm{CH}_{3}\right)_{3} \mathrm{CF}_{3}$}

$1 \backslash 1 \backslash G I N C-S C 96 \backslash F O p t \backslash R B 3 L Y P \backslash 6-31 G(d) \backslash C 4 H 10 F 3 P 1 \backslash J O H 501 \backslash 15-D e c-2004 \backslash 0 \backslash \backslash \# B$ 3LYP/6-31G* OPT FREQ MAXDISK=134217728\\a_pme3cf3_ax32 opt freq b3lyp \ $\backslash 0,1 \backslash \mathrm{P}, 0.8084681666,-0.2150880906,0 . \backslash \mathrm{H}, 0 . \overline{5} 3631218 \overline{8} 9,-1.6222816207,0 . \backslash \mathrm{C}$ $,-1.1900606498,-0.1021461902,0 . \backslash \mathrm{F},-1.7350917946,1.153906131,0 . \backslash \mathrm{C}, 2.683$ $2215519,-0.6324986832,0 . \backslash \mathrm{H}, 3.3375043546,0.2483540684,0 . \backslash \mathrm{F},-1.703751614$ $4,-0.7402762782,1.090941417 \backslash \mathrm{F},-1.7037516144,-0.7402762782,-1.090941417$ $\backslash \mathrm{C}, 0.8578734355,0.7434329317,1.5905342111 \backslash \mathrm{C}, 0.8578734355,0.7434329317$, $-1.5905342111 \backslash \mathrm{H}, 0.0614899687,1.4893405671,1.6162746008 \backslash \mathrm{H}, 0.0614899687$, $1.4893405671,-1.6162746008 \backslash \mathrm{H}, 0.6995836553,0.0427447949,2.4163854734 \backslash \mathrm{H}$, $0.6995836553,0.0427447949,-2.4163854734 \backslash \mathrm{H}, 1.8328252086,1.2188349638,1$. $7155993366 \backslash \mathrm{H}, 1.8328252086,1.2188349638,-1.7155993366 \backslash \mathrm{H}, 2.9206359317,-1$ $.2375499259,0.8852439458 \backslash \mathrm{H}, 2.9206359317,-1.2375499259,-0.8852439458 \backslash \backslash \mathrm{V}$ ersion=DEC-AXP-OSF $/ 1-G 03 R e v B .03 \backslash$ State $=1-A^{\prime} \backslash H F=-799.26581 \backslash R M S D=3.398 e-0$ $9 \backslash \mathrm{RMSF}=3.783 e-05 \backslash \mathrm{Dipole}=1.0977359,0.30576,0 . \backslash \mathrm{PG}=\mathrm{CS} \quad[\mathrm{SG}(\mathrm{C} 2 \mathrm{H} 2 \mathrm{~F} 1 \mathrm{P} 1), \mathrm{X}(\mathrm{C} 2 \mathrm{H}$ $8 \mathrm{~F} 2)] \backslash \backslash[$

\section{Equatorial $\cdot \mathrm{P}\left(\mathrm{CH}_{3}\right)_{3} \mathrm{CF}_{3}(15)$}

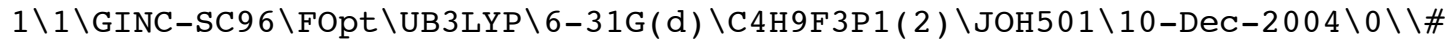
B3LYP/6-31G* OPT FREQ MAXDISK=134217728\\pme3cf3_eq2 opt freq b3lyp $\backslash \backslash$ $0,2 \backslash \mathrm{C},-2.0480233355,0.577909701,-0.8487381977 \backslash \mathrm{P},-0.0583288205,0.496603$ $8469,-0.814916667 \backslash \mathrm{C}, 1.8728413119,1.0038609146,-0.7705164603 \backslash \mathrm{C}, 0.023433$ $0971,-0.2132636449,0.9511014906 \backslash \mathrm{C}, 0.0928066966,-1.0383592275,-1.849254$ $5592 \backslash \mathrm{H},-0.4910076025,-1.855145359,-1.4140883456 \backslash \mathrm{H}, 1.133909222,-1.35444$ $80853,-1.9426523652 \backslash \mathrm{H},-0.3057218717,-0.8318494859,-2.8477910862 \backslash \mathrm{H},-2.5$ $111453523,-0.4019241703,-0.6913826246 \backslash \mathrm{H},-2.3787034047,1.2751976605,-0$. $0740500611 \backslash \mathrm{H},-2.3211374033,0.978104056,-1.8293895171 \backslash \mathrm{H}, 2.5093034591,0$. $1758465229,-0.441402952 \backslash \mathrm{H}, 2.133528435,1.3093643649,-1.7878457476 \backslash \mathrm{H}, 1.9$ $860505589,1.8574545006,-0.0973517142 \backslash \mathrm{F}, 1.0497263715,-1.0841519971,1.10$ $46381616 \backslash \mathrm{F}, 0.2089737492,0.7846911374,1.8480677024 \backslash \mathrm{F},-1.0949768267,-0.8$ $763773809,1.3199775559 \backslash \backslash$ Version=DEC-AXP-OSF / 1-G03RevB.03 $\backslash$ State $=2-\mathrm{A} \backslash \mathrm{HF}=$ $-798.6462495 \backslash \mathrm{S} 2=0.755305 \backslash \mathrm{S} 2-1=0 . \backslash \mathrm{S} 2 \mathrm{~A}=0.75002 \backslash \mathrm{RMSD}=8.372 \mathrm{e}-09 \backslash \mathrm{RMSF}=5.114$ e-06\Dipole $=-0.0205086,-0.1121503,-0.7789117 \backslash \mathrm{PG}=\mathrm{C} 01 \quad[\mathrm{X}(\mathrm{C} 4 \mathrm{H} 9 \mathrm{~F} 3 \mathrm{P} 1)] \backslash \backslash @$

\section{$\mathrm{P}\left(\mathrm{CH}_{3}\right)_{3}$}

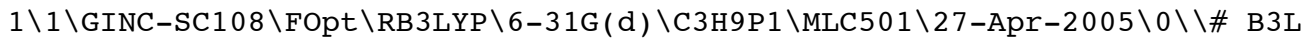




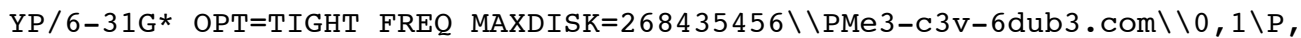
$0 ., 0 ., 0.6052805807 \backslash \mathrm{C}, 1.6439649058,0 .,-0.280577585 \backslash \mathrm{C},-0.8219824529,-1.4$ $237153714,-0.280577585 \backslash \mathrm{C},-0.8219824529,1.4237153714,-0.280577585 \backslash \mathrm{H}, 1.5$ $368236996,0 .,-1.3726206461 \backslash \mathrm{H},-0.7684118498,-1.330928365,-1.3726206461 \backslash$ $\mathrm{H},-0.7684118498,1.330928365,-1.3726206461 \backslash \mathrm{H}, 2.2199394809,0.8837410205$, $0.0148416263 \backslash \mathrm{H},-0.3446275663,-2.3643944955,0.0148416263 \backslash \mathrm{H},-1.875311914$ $5,1.480653475,0.0148416263 \backslash \mathrm{H}, 2.2199394809,-0.8837410205,0.0148416263 \backslash \mathrm{H}$ $,-1.8753119145,-1.480653475,0.0148416263 \backslash \mathrm{H},-0.3446275663,2.3643944955$, $0.0148416263 \backslash \backslash$ Version $=\mathrm{A} 164 \mathrm{~T}-\mathrm{G} 03$ RevC $.02 \backslash$ State $=1-\mathrm{A} 1 \backslash \mathrm{HF}=-461.0984306 \backslash \mathrm{RMSD}$ $=9.936 \mathrm{e}-09 \backslash \mathrm{RMSF}=1.057 \mathrm{e}-06 \backslash \mathrm{Dipole}=0 ., 0 .,-0.5305947 \backslash \mathrm{PG}=\mathrm{C} 03 \mathrm{~V} \quad[\mathrm{C} 3(\mathrm{P} 1), 3 \mathrm{SGV}$ $(\mathrm{C} 1 \mathrm{H} 1), \mathrm{x}(\mathrm{H} 6) \mathrm{]} \backslash \backslash \mathrm{Q}$

\section{$\cdot \mathrm{CH}_{3}$}

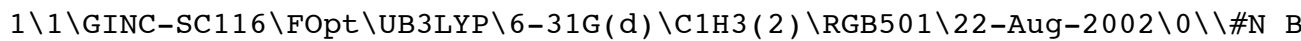
3LYP 6-31G(D) SCF=TIGHT OPT=TIGHT FREQ MAXDISK=13107200 \methyl Opt\&Fr eq UB3-LYP $/ 6-31 \mathrm{G}(\mathrm{d}) \backslash \backslash 0,2 \backslash \mathrm{C}, 0 ., 0 ., 0 . \backslash \mathrm{H}, 1.082752351,0 ., 0 . \backslash \mathrm{H},-0.541376175$ $5,-0.937691042,0 . \backslash \mathrm{H},-0.5413761755,0.937691042,0 . \backslash \backslash$ Version=DEC-AXP-OSF / 1-G98RevA. $11.3 \backslash \mathrm{HF}=-39.8382922 \backslash \mathrm{S} 2=0.753765 \backslash \mathrm{S} 2-1=0 . \backslash \mathrm{S} 2 \mathrm{~A}=0.750007 \backslash \mathrm{RMSD}=4$. $410 \mathrm{e}-09 \backslash \mathrm{RMSF}=1.426 \mathrm{e}-09 \backslash \mathrm{Dipole}=0 ., 0 ., 0 . \backslash \mathrm{PG}=\mathrm{D} 03 \mathrm{H} \quad[\mathrm{O}(\mathrm{C} 1), 3 \mathrm{C} 2(\mathrm{H} 1)] \backslash \backslash @$

\section{$\mathrm{P}\left(\mathrm{CH}_{3}\right)_{2} \mathrm{CF}_{3}$}

$1 \backslash 1 \backslash$ GINC-AC3 $\$ FOpt $\backslash R B 3 L Y P \backslash 6-31 G(d) \backslash C 3 H 6 F 3 P 1 \backslash M L C 501 \backslash 08-J u n-2005 \backslash 0 \backslash \backslash \#$ B3L

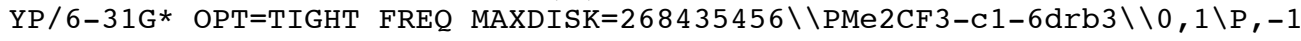
$.104140775,-0.2381556392,0 . \backslash \mathrm{C}, 0.7788263636,-0.3977755823,0 . \backslash \mathrm{F}, 1.446134$ $1037,0.7842599557,0 . \backslash \mathrm{F}, 1.1932185419,-1.0795922237,-1.0920347673 \backslash \mathrm{F}, 1.19$ $32185419,-1.0795922237,1.0920347673 \backslash \mathrm{C},-1.2411595394,0.9425768225,-1.43$ $53994615 \backslash \mathrm{C},-1.2411595394,0.9425768225,1.4353994615 \backslash \mathrm{H},-0.5855383126,1.8$ $124045466,-1.3287900542 \backslash \mathrm{H},-0.5855383126,1.8124045466,1.3287900542 \backslash \mathrm{H},-0$ $.9902729902,0.4167917808,-2.3617287225 \backslash \mathrm{H},-0.9902729902,0.4167917808,2$. $3617287225 \backslash \mathrm{H},-2.2792250831,1.281996991,-1.5112141011 \backslash \mathrm{H},-2.2792250831,1$ $.281996991,1.5112141011 \backslash \backslash$ Version=IA64L-G03RevC.02 $\backslash$ State $=1-A^{\prime} \backslash \mathrm{HF}=-758.8$ $100768 \backslash \mathrm{RMSD}=2.734 \mathrm{e}-09 \backslash \mathrm{RMSF}=1.120 \mathrm{e}-06 \backslash \mathrm{Dipole}=-0.6369525,0.6864918,0 . \backslash \mathrm{PG}$ $=\mathrm{CS}[\mathrm{SG}(\mathrm{C} 1 \mathrm{~F} 1 \mathrm{P} 1), \mathrm{X}(\mathrm{C} 2 \mathrm{H} 6 \mathrm{~F} 2)] \backslash \backslash @$

\section{$\cdot \mathrm{CF}_{3}$}

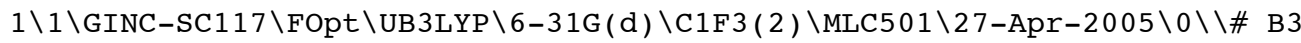

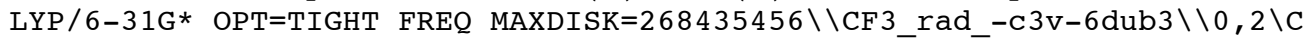
$, 0.0 .0 .3288690179 \backslash \mathrm{F}, 1.2645504969,0 \ldots,-0.073082004 \backslash \mathrm{F},-0.6322752484,-1$. $0951328547,-0.073082004 \backslash \mathrm{F},-0.6322752484,1.0951328547,-0.073082004 \backslash \backslash$ Ver sion $=\mathrm{A} 164 \mathrm{~T}-\mathrm{G} 03 \mathrm{RevC} .02 \backslash$ State $=2-\mathrm{A} 1 \backslash \mathrm{HF}=-337.5509952 \backslash \mathrm{S} 2=0.751413 \backslash \mathrm{S} 2-1=0 . \backslash \mathrm{S}$ $2 \mathrm{~A}=0.750001 \backslash \mathrm{RMSD}=2.911 \mathrm{e}-09 \backslash \mathrm{RMSF}=3.277 \mathrm{e}-08 \backslash \mathrm{Dipole}=0,0 ., 0.0871342 \backslash \mathrm{PG}=\mathrm{C} 0$ $3 \mathrm{~V}[\mathrm{C} 3(\mathrm{C} 1), 3 \mathrm{SGV}(\mathrm{F} 1)] \backslash \backslash @$

\section{$\mathrm{P}\left(\mathrm{CH}_{3}\right)_{2} \mathrm{CN}$}

$1 \backslash 1 \backslash G I N C-A C 3 \backslash F O p t \backslash R B 3 L Y P \backslash 6-31 G(d) \backslash C 3 H 6 N 1 P 1 \backslash M L C 501 \backslash 08-J u n-2005 \backslash 0 \backslash \backslash \#$ B3L

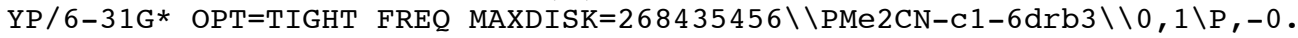
$6338171761,0.369987373,0 . \backslash \mathrm{C},-0.0095353399,-1.3198938469,0 . \backslash \mathrm{N}, 0.3060963$ $809,-2.4410334621,0 . \backslash \mathrm{C}, 0.3528267819,1.0322139252,1.4395291694 \backslash \mathrm{C}, 0.3528$ $267819,1.0322139252,-1.4395291694 \backslash \mathrm{H}, 0.1642281896,2.1087974932,1.510612$ $9252 \backslash \mathrm{H}, 0.1642281896,2.1087974932,-1.5106129252 \backslash \mathrm{H}, 0.0015475768,0.565559$ $1914,2.3642902129 \backslash \mathrm{H}, 0.0015475768,0.5655591914,-2.3642902129 \backslash \mathrm{H}, 1.428161$ $0495,0.8607531251,1.3361016148 \backslash \mathrm{H}, 1.4281610495,0.8607531251,-1.33610161$

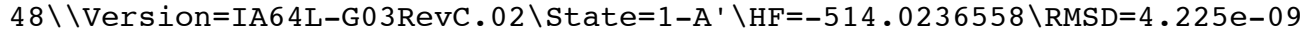
$\backslash \mathrm{RMSF}=1.050 \mathrm{e}-06 \backslash \mathrm{Dipole}=0.1858773,1.6022863,0 . \backslash \mathrm{PG}=\mathrm{CS} \quad[\mathrm{SG}(\mathrm{C} 1 \mathrm{~N} 1 \mathrm{P} 1), \mathrm{X}(\mathrm{C} 2 \mathrm{H} 6$ )$] \backslash \backslash @$

\section{$\cdot \mathrm{CN}$}

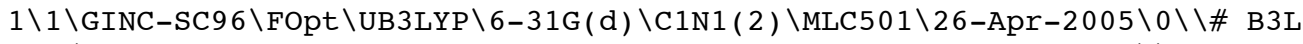
YP/6-31G* OPT=TIGHT FREQ MAXDISK=134217728 MAXDISK=134217728 \\CN_rad-6 dub3 $\backslash \backslash 0,2 \backslash C,-0.6321098425,-0.0000161811,0 . \backslash N, 0.5418084364,0.0000 \overline{1} 38695$ , $0 . \backslash$ Version=Al64T-G03RevC.02\State=2-SG $\backslash \mathrm{HF}=-92.7117473 \backslash \mathrm{S} 2=0.75741 \backslash \mathrm{S} 2-$ 
$1=0 . \backslash \mathrm{S} 2 \mathrm{~A}=0.750041 \backslash \mathrm{RMSD}=1.173 \mathrm{e}-09 \backslash \mathrm{RMSF}=3.051 \mathrm{e}-11 \backslash \mathrm{Dipole}=-0.4988141,-0.0$ $000128,0 . \backslash P G=C * V[C *(C 1 N 1)] \backslash \backslash @$

\section{$\mathrm{P}\left(\mathrm{CH}_{3}\right)_{2} \mathrm{~F}$}

$1 \backslash 1 \backslash$ GINC-AC3 $\backslash$ FOpt $\backslash R B 3 L Y P \backslash 6-31 G(d) \backslash C 2 H 6 F 1 P 1 \backslash M L C 501 \backslash 08-J u n-2005 \backslash 0 \backslash \backslash \#$ B3L

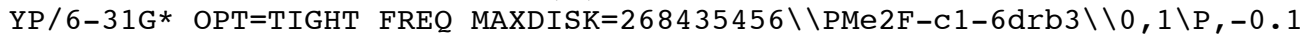
$144324876,-0.5683618406,0 . \backslash \mathrm{C}, 0.7706492459,0.2500740325,1.4075074223 \backslash \mathrm{C}$, $0.7706492459,0.2500740325,-1.4075074223 \backslash \mathrm{F},-1.4748973667,0.3455622263,0$ . \H, $1.7927222031,-0.1433917306,-1.4661124217 \backslash \mathrm{H}, 1.7927222031,-0.1433917$ $306,1.4661124217 \backslash \mathrm{H}, 0.2657690809,0.0114359798,-2.3491228943 \backslash \mathrm{H}, 0.2657690$ $809,0.0114359798,2.3491228943 \backslash \mathrm{H}, 0.8128950483,1.3391953425,-1.293831437$ $\backslash \mathrm{H}, 0.8128950483,1.3391953425,1.293831437 \backslash \backslash$ Version=IA64L-G03RevC.02\Sta $t e=1-A^{\prime} \backslash H F=-521.044039 \backslash R M S D=9.382 e-09 \backslash R M S F=5.514 e-06 \backslash D i p o l e=0.7763317$, $0.177991,0 . \backslash P G=C S[S G(F 1 P 1), X(C 2 H 6)] \backslash \backslash @$

$\bullet \mathbf{F}$

$1 \backslash 1 \backslash G I N C-S C 103 \backslash S P \backslash U B 3 L Y P \backslash 6-31 G(d) \backslash F 1(2) \backslash M L C 501 \backslash 12-A u g-2003 \backslash 0 \backslash \backslash \#$ B 3LYP / $6-31 \mathrm{G} * \mathrm{SCF}=\mathrm{TIGHT}$ MAXDISK $=134217728 \backslash \backslash \mathrm{F}$ atom $\backslash \backslash 0,2 \backslash \mathrm{F} \backslash \backslash$ Version=DEC-AXP-OSF $/ 1-\mathrm{G} 03 \mathrm{RevB} .03 \backslash \mathrm{HF}=-99.7155365 \backslash \mathrm{S} 2=0.750978 \backslash \mathrm{S} 2-1=0 . \backslash \mathrm{S} 2 \mathrm{~A}=0.75 \backslash \mathrm{RMSD}=1.596 \mathrm{e}-$ $09 \backslash \mathrm{Dipole}=0 ., 0 ., 0 . \backslash \mathrm{PG}=\mathrm{OH}[\mathrm{O}(\mathrm{F} 1)] \backslash \backslash @$

\section{$\mathrm{P}\left(\mathrm{CH}_{3}\right)_{2} \mathrm{OH}$}

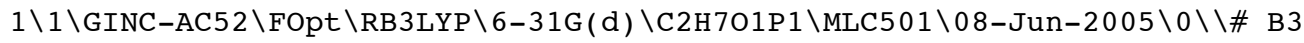
LYP $/ 6-31 \mathrm{G} *$ OPT=TIGHT FREQ MAXDISK $=65536000$ MAXDISK $=65536000 \backslash \backslash \mathrm{PMe} 2 \mathrm{OH}-\mathrm{CS}$ $-6 \mathrm{drb} 3 \backslash \backslash 0,1 \backslash \mathrm{P}, 0.3527495668,0.444096652,0 . \backslash 0,-1.2909005184,0.8455845695$ $, 0 . \backslash \mathrm{H},-1.3710214822,1.8105203128,0 . \backslash \mathrm{C}, 0.3527798181,-0.7657806473,1.406$ $9404114 \backslash \mathrm{C}, 0.3527798181,-0.7657806473,-1.4069404114 \backslash \mathrm{H}, 1.3088188554,-1.3$ $021621193,1.4314259859 \backslash \mathrm{H}, 1.3088188554,-1.3021621193,-1.4314259859 \backslash \mathrm{H}, 0$. $2407946483,-0.2292671906,2.3544810705 \backslash \mathrm{H}, 0.2407946483,-0.2292671906,-2$. $3544810705 \backslash \mathrm{H},-0.4628013481,-1.492210131,1.3142606787 \backslash \mathrm{H},-0.4628013481,-$ $1.492210131,-1.3142606787 \backslash \backslash$ Version=IA64L-G03RevC .02 $\backslash$ State $=1-A^{\prime} \backslash \mathrm{HF}=-497$ $.0138502 \backslash \mathrm{RMSD}=3.501 \mathrm{e}-09 \backslash \mathrm{RMSF}=6.366 \mathrm{e}-07 \backslash \mathrm{Dipole}=0.114156,0.0089943,0 . \backslash \mathrm{PG}$ $=\mathrm{CS} \quad[\mathrm{SG}(\mathrm{H} 101 \mathrm{P} 1), \mathrm{X}(\mathrm{C} 2 \mathrm{H} 6)] \backslash \backslash @$

\section{$\cdot \mathrm{OH}$}

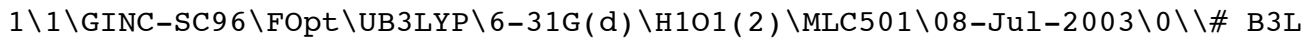

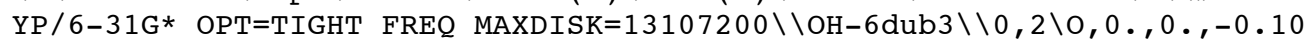
$9212493 \backslash \mathrm{H}, 0.0 .0 .8736999437 \backslash \backslash$ Version=DEC-AXP-OSF $/ 1-G 03$ RevB . 03 $\backslash \mathrm{HF}=-75$. $7234548 \backslash \mathrm{S} 2=0.751967 \backslash \mathrm{S} 2-1=0 . \backslash \mathrm{S} 2 \mathrm{~A}=0.750002 \backslash \mathrm{RMSD}=1.307 \mathrm{e}-09 \backslash \mathrm{RMSF}=1.522 \mathrm{e}-08$ $\backslash$ Dipole $=0 ., 0 ., 0.6914207 \backslash P G=C * V \quad[C *(H 101)] \backslash \backslash @$

\section{$\mathrm{P}\left(\mathrm{CH}_{3}\right)_{2} \mathrm{OCH}_{3}$}

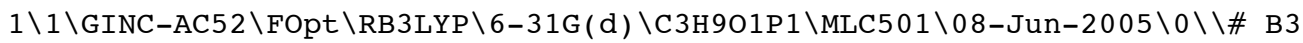

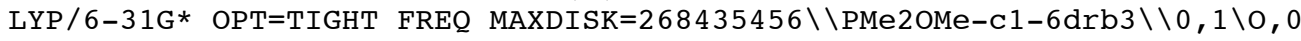
$.9559509373,-0.3317161806,0.480282 \backslash P,-0.342684002,-0.0107619353,-0.547$ $594 \backslash \mathrm{C},-1.5137382334,-1.235215714,0.203939 \backslash \mathrm{C},-1.0161417092,1.539471192$, $0.23454 \backslash \mathrm{H},-1.1316227293,1.4330092138,1.319178 \backslash \mathrm{H},-1.9898406635,1.781099$ $3759,-0.20891 \backslash \mathrm{H},-0.3422245506,2.3787430647,0.031786 \backslash \mathrm{H},-1.5218912192,-1$ $.1601527125,1.297052 \backslash \mathrm{H},-1.217018425,-2.2496897701,-0.079135 \backslash \mathrm{H},-2.52570$ $81993,-1.0550015228,-0.176688 \backslash \mathrm{C}, 2.2592499926,-0.0390094268,-0.010845 \backslash \mathrm{H}$ $, 2.9722419028,-0.5147335615,0.669144 \backslash \mathrm{H}, 2.4146149184,-0.4318774562,-1.0$ $24543 \backslash \mathrm{H}, 2.4578871969,1.0422825357,-0.022034 \backslash \backslash$ Version=IA64L-G03RevC .02 State $=1-\mathrm{A} \backslash \mathrm{HF}=-536.3176838 \backslash \mathrm{RMSD}=2.932 \mathrm{e}-09 \backslash \mathrm{RMSF}=3.193 \mathrm{e}-06 \backslash \mathrm{Dipole}=-0.0538$ $258,0.1849306,0.0510003 \backslash \mathrm{PG}=\mathrm{C} 01[\mathrm{X}(\mathrm{C} 3 \mathrm{H} 901 \mathrm{P} 1)] \backslash \backslash @$

\section{$\cdot \mathrm{OCH}_{3}$}

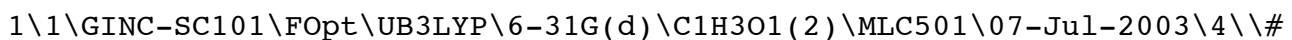

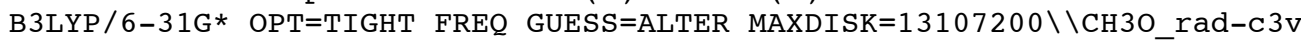
-6 dub3 $\backslash \backslash 0,2 \backslash 0,-0.0717954037,0.7904851464,0 . \backslash C, 0.070527331,-0.5 \overline{7} 0652403$ $1,0 . \backslash \mathrm{H},-0.9643070065,-0.9734523539,0 . \backslash \mathrm{H}, 0.557753125,-0.9632571993,0.90$ 
$88020881 \backslash \mathrm{H}, 0.557753125,-0.9632571993,-0.9088020881 \backslash \backslash \backslash 8,9 \backslash \backslash$ Version=DECAXP-OSF $/ 1-G 03 R e v B .03 \backslash S_{\text {tate }}=2-A^{\prime} \backslash H F=-115.0504626 \backslash S 2=0.752916 \backslash \mathrm{S} 2-1=0 . \backslash S 2$ $\mathrm{A}=0.750006 \backslash \mathrm{RMSD}=4.434 \mathrm{e}-09 \backslash \mathrm{RMSF}=5.514 \mathrm{e}-06 \backslash \mathrm{Dipole}=-0.0146059,-0.780437,0$ $. \backslash P G=C S[S G(C 1 H 1 O 1), X(H 2)] \backslash \backslash @$

\section{$\mathrm{P}\left(\mathrm{CH}_{3}\right)_{2} \mathrm{Ph}$}

$1 \backslash 1 \backslash$ GINC-AC52 $\backslash$ FOpt $\backslash R B 3 L Y P \backslash 6-31 G(d) \backslash C 8 H 11 P 1 \backslash M L C 501 \backslash 08-J u n-2005 \backslash 0 \backslash \backslash \#$ B $3 L$

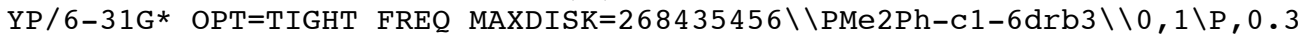
$54889,-1.729056,0 . \backslash \mathrm{C}, 0.235692,0.119577,0 . \backslash \mathrm{C}, 0.243353,2.944917,0 . \backslash \mathrm{H}, 0.2$ $42631,4.031583,0 . \backslash \mathrm{C},-0.778882,-2.167983,1.418976 \backslash \mathrm{C},-0.778882,-2.167983$ $,-1.418976 \backslash C, 0.245892,0.844715,1.203679 \backslash C, 0.245892,0.844715,-1.203679 \backslash$ $\mathrm{C}, 0.245892,2.240055,1.204399 \backslash \mathrm{C}, 0.245892,2.240055,-1.204399 \backslash \mathrm{H},-0.95675$, $-3.248625,1.391301 \backslash \mathrm{H},-0.95675,-3.248625,-1.391301 \backslash \mathrm{H},-0.297386,-1.94373$ $7,2.375443 \backslash \mathrm{H},-0.297386,-1.943737,-2.375443 \backslash \mathrm{H},-1.741803,-1.647042,1.374$ $723 \backslash \mathrm{H},-1.741803,-1.647042,-1.374723 \backslash \mathrm{H}, 0.251847,0.320849,2.155711 \backslash \mathrm{H}, 0.2$ $51847,0.320849,-2.155711 \backslash \mathrm{H}, 0.246568,2.77648,2.150053 \backslash \mathrm{H}, 0.246568,2.7764$ $8,-2.150053 \backslash \backslash$ Version $=I A 64 L-G 03 R e v C .02 \backslash$ State $^{\prime}=1-A^{\prime} \backslash \mathrm{HF}=-652.8299923 \backslash \mathrm{RMSD}=$ $4.569 \mathrm{e}-09 \backslash \mathrm{RMSF}=4.925 \mathrm{e}-07 \backslash \mathrm{Dipole}=-0.5268215,0.0789726,0 . \backslash \mathrm{PG}=\mathrm{CS} \quad[\mathrm{SG}(\mathrm{C} 2 \mathrm{H} 1$ $\mathrm{P} 1), \mathrm{X}(\mathrm{C} 6 \mathrm{H} 10)] \backslash \backslash @$

\section{$\cdot \mathrm{Ph}$}

$1 \backslash 1 \backslash G I N C-S C 90 \backslash F O p t \backslash U B 3 L Y P \backslash 6-31 G(d) \backslash C 6 H 5(2) \backslash M L C 501 \backslash 26-A p r-2005 \backslash 0 \backslash \backslash \#$ B3L YP/6-31G* OPT=TIGHT FREQ MAXDISK $=134217728 \backslash \backslash \mathrm{Ph}$ rad-6dub3 $\backslash \backslash 0,2 \backslash \mathrm{C}, 0 ., 0$, , $1.4003036877 \backslash \mathrm{C}, 0 ., 1.2262305038,0.7718051476 \backslash \mathrm{C}, \overline{0} .,-1.2262305038,0.77180$ $51476 \backslash C, 0 ., 1.2141380645,-0.6326273883 \backslash C, 0 .,-1.2141380645,-0.6326273883$ $\backslash \mathrm{C}, 0 ., 0 .,-1.3247593826 \backslash \mathrm{H}, 0 ., 2.1628282348,1.323175196 \backslash \mathrm{H}, 0 .,-2.162828234$ $8,1.323175196 \backslash \mathrm{H}, 0$. 2. $2.1545167594,-1.1791862459 \backslash \mathrm{H}, 0$. , -2 .1545167594, -1.17 $91862459 \backslash \mathrm{H}, 0 ., 0 .,-2.4113768423 \backslash \backslash$ Version=Al64 T-G03RevC.02 $\backslash$ State $=2-\mathrm{A} 1 \backslash \mathrm{HF}$ $=-231.5612809 \backslash \mathrm{S} 2=0.75748 \backslash \mathrm{S} 2-1=0 . \backslash \mathrm{S} 2 \mathrm{~A}=0.750031 \backslash \mathrm{RMSD}=7.315 \mathrm{e}-09 \backslash \mathrm{RMSF}=1.20$ $6 \mathrm{e}-06 \backslash \mathrm{Dipole}=0 ., 0 .,-0.3087677 \backslash \mathrm{PG}=\mathrm{C} 02 \mathrm{~V} \quad[\mathrm{C} 2(\mathrm{H} 1 \mathrm{C} 1 \mathrm{C} 1), \mathrm{SGV}(\mathrm{C} 4 \mathrm{H} 4)] \backslash \backslash @$

\section{$\mathrm{P}\left(\mathrm{CH}_{3}\right)_{2} \mathrm{SCH}_{3}$}

$1 \backslash 1 \backslash$ GINC-AC2 $\backslash$ FOpt $\backslash R B 3 L Y P \backslash 6-31 G(d) \backslash C 3 H 9 P 1 S 1 \backslash M L C 501 \backslash 08-J u n-2005 \backslash 0 \backslash \backslash \#$ B3L YP $/ 6-31 \mathrm{G} *$ OPT $=$ TIGHT FREQ MAXDISK $=268435456$ MAXDISK $=268435456 \backslash \backslash \mathrm{PMe} 2 \mathrm{SCH} 3$ $-\mathrm{c} 1-6 \mathrm{drb} 3 \backslash \backslash 0,1 \backslash \mathrm{S},-1.2652304355,-0.2031338869,-0.0959299892 \backslash \mathrm{P}, 0.8625410$ $663,0.0293125485,0.1624343442 \backslash \mathrm{C}, 0.9628155255,1.8675539549,0.4748824774$ $\backslash \mathrm{C}, 1.4201215538,0.0107820123,-1.6195830399 \backslash \mathrm{H}, 1.3102249783,-0.999415211$ $6,-2.0241037359 \backslash \mathrm{H}, 2.4820027773,0.2823557554,-1.6576668354 \backslash \mathrm{H}, 0.85533427$ $12,0.7067203807,-2.2492028091 \backslash \mathrm{H}, 2.0177415629,2.1673581279,0.4685116134$ $\backslash \mathrm{H}, 0.424301149,2.4564893561,-0.2753806727 \backslash \mathrm{H}, 0.5491104908,2.0912870002$, $1.4623045403 \backslash \mathrm{C},-1.5955563878,-1.5930018956,1.0631450962 \backslash \mathrm{H},-2.669233511$ $,-1.7906738113,1.0041674524 \backslash \mathrm{H},-1.0501954651,-2.4925272793,0.7694399053$ $\backslash \mathrm{H},-1.3379994287,-1.3231447841,2.0896280049 \backslash \backslash$ Version=IA64L-G03RevC.02 State $=1-\mathrm{A} \backslash \mathrm{HF}=-859.2999066 \backslash \mathrm{RMSD}=2.209 \mathrm{e}-09 \backslash \mathrm{RMSF}=8.581 \mathrm{e}-07 \backslash \mathrm{Dipole}=0.33642$ $24,0.0311543,0.045536 \backslash \mathrm{PG}=\mathrm{C} 01[\mathrm{X}(\mathrm{C} 3 \mathrm{H} 9 \mathrm{P} 1 \mathrm{~S} 1)] \backslash \backslash @$

\section{$\cdot \mathrm{SCH}_{3}$}

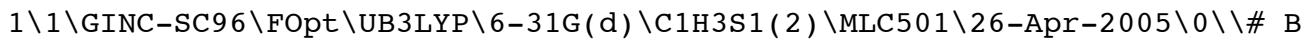
3LYP/6-31G* OPT=TIGHT FREQ MAXDISK=134217728 MAXDISK=134217728\\CH3S_r ad-6dub3.com $\backslash \backslash 0,2 \backslash C,-0.0015256918,1.1151272943,0 . \backslash S,-0.0059130885,-0.6$ $976226135,0 . \backslash \mathrm{H}, 1.051045679,1.4307045833,0 . \backslash \mathrm{H},-0.4736410562,1.520246733$ $5,0.8993258184 \backslash \mathrm{H},-0.4736410562,1.5202467335,-0.8993258184 \backslash \backslash$ Version=Al 6 $4 \mathrm{~T}-\mathrm{G} 03$ RevC.02 $\backslash$ State $=2-\mathrm{A}^{\prime} \backslash \mathrm{HF}=-438.0597247 \backslash \mathrm{S} 2=0.752489 \backslash \mathrm{S} 2-1=0 . \backslash \mathrm{S} 2 \mathrm{~A}=0.750$ $003 \backslash \mathrm{RMSD}=7.235 \mathrm{e}-09 \backslash \mathrm{RMSF}=4.019 \mathrm{e}-06 \backslash \mathrm{Dipole}=0.0385923,0.7049947,0 . \backslash \mathrm{PG}=\mathrm{CS}$ [SG(C1H1S1),X(H2)]\\@

\section{$\left(\mathrm{CH}_{3}\right)_{2} \mathrm{C} \cdot \mathrm{CF}_{3}$}

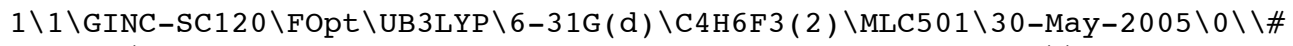

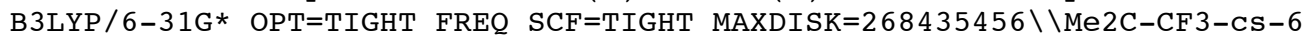
$\mathrm{drb} 3 \backslash \backslash 0,2 \backslash \mathrm{C}, 0.1556956552,-0.8502832955,0 . \backslash \mathrm{C}, 0.0237687854,0.6333310138$, $0 . \backslash C, 0.0064101582,-1.5753899628,-1.2981198126 \backslash \mathrm{C}, 0.0064101582,-1.575389$ 
$9628,1.2981198126 \backslash \mathrm{F},-1.2839360358,1.0294310778,0 . \backslash \mathrm{H},-1.0518999538,-1.8$ $050249746,-1.511628149 \backslash \mathrm{H},-1.0518999538,-1.8050249746,1.511628149 \backslash \mathrm{F}, 0.5$ $920292615,1.1998123775,1.0904880416 \backslash \mathrm{F}, 0.5920292615,1.1998123775,-1.090$ $4880416 \backslash \mathrm{H}, 0.3873300074,-0.9882171287,-2.1379533041 \backslash \mathrm{H}, 0.5371644827,-2.5$ $343125229,-1.2702258982 \backslash \mathrm{H}, 0.5371644827,-2.5343125229,1.2702258982 \backslash \mathrm{H}, 0$. $3873300074,-0.9882171287,2.1379533041 \backslash \backslash$ Version=Al64T-G03RevC.02 $\backslash$ State $=$ $2-\mathrm{A}^{\prime} \backslash \mathrm{HF}=-455.5214692 \backslash \mathrm{S} 2=0.753691 \backslash \mathrm{S} 2-1=0 . \backslash \mathrm{S} 2 \mathrm{~A}=0.75001 \backslash \mathrm{RMSD}=3.294 \mathrm{e}-09 \backslash \mathrm{RM}$ $\mathrm{SF}=1.670 \mathrm{e}-06 \backslash \mathrm{Dipole}=0.0011564,-0.942763,0 . \backslash \mathrm{PG}=\mathrm{CS}[\mathrm{SG}(\mathrm{C} 2 \mathrm{~F} 1), \mathrm{X}(\mathrm{C} 2 \mathrm{H} 6 \mathrm{~F} 2)] \backslash$ $\backslash @$

\section{$\left(\mathrm{CH}_{3}\right)_{2} \mathrm{CHCF}_{3}$}

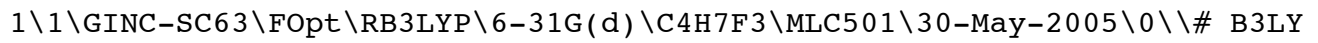
P/6-31G* OPT=TIGHT FREQ SCF=TIGHT MAXDISK=268435456\\Me2CH-CF3-CS-6drb $3 \backslash \backslash 0,1 \backslash \mathrm{C}, 0.4683501312,-0.8084617944,0 . \backslash \mathrm{H}, 1.5649157795,-0.7615830717,0$. $\backslash \mathrm{C}, 0.0118842904,0.6420641318,0 . \backslash \mathrm{C},-0.0017666192,-1.5234006308,-1.27440$ $24393 \backslash \mathrm{C},-0.0017666192,-1.5234006308,1.2744024393 \backslash \mathrm{F},-1.3385037076,0.748$ $5833737,0 . \backslash \mathrm{H},-1.0954743947,-1.5647550818,-1.3151182623 \backslash \mathrm{H},-1.0954743947$ $,-1.5647550818,1.3151182623 \backslash \mathrm{F}, 0.4636435985,1.3088412007,1.0878356886 \backslash \mathrm{F}$ , $0.4636435985,1.3088412007,-1.0878356886 \backslash \mathrm{H}, 0.3553481379,-1.0132199689$, $-2.1736365496 \backslash \mathrm{H}, 0.3780391157,-2.5498326288,-1.2886399848 \backslash \mathrm{H}, 0.378039115$ $7,-2.5498326288,1.2886399848 \backslash \mathrm{H}, 0.3553481379,-1.0132199689,2.1736365496$ $\backslash \backslash$ Version=Al64T-G03RevC.02 $\backslash$ State $=1-A^{\prime} \backslash \mathrm{HF}=-456.1850437 \backslash \mathrm{RMSD}=5.858 \mathrm{e}-09 \backslash \mathrm{R}$ $\mathrm{MSF}=6.587 \mathrm{e}-06 \backslash \mathrm{Dipole}=0.2174639,-0.7978654,0 . \backslash \mathrm{PG}=\mathrm{CS} \quad[\mathrm{SG}(\mathrm{C} 2 \mathrm{H} 1 \mathrm{~F} 1), \mathrm{X}(\mathrm{C} 2 \mathrm{H} 6 \mathrm{~F}$ 2) $] \backslash \backslash @$

\section{$\left(\mathrm{CH}_{3}\right)_{2} \mathrm{C} \cdot \mathrm{F}$}

$1 \backslash 1 \backslash G I N C-S C 122 \backslash F O p t \backslash U B 3 L Y P \backslash 6-31 G(d) \backslash C 3 H 6 F 1(2) \backslash M L C 501 \backslash 30-M a y-2005 \backslash 0 \backslash \backslash \#$

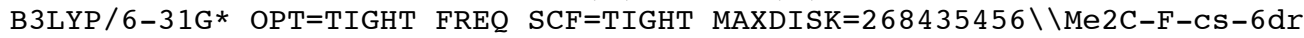
b3 $\backslash \backslash 0,2 \backslash C, 0.1727227569,0.1448129571,0 . \backslash F,-0.8189996115,1.0849254892,0$. $\backslash \mathrm{C}, 0.3378587577,-0.5308213579,1.3165370899 \backslash \mathrm{C}, 0.3378587577,-0.530821357$ $9,-1.3165370899 \backslash \mathrm{H}, 0.4009518879,0.2056583083,2.1257268264 \backslash \mathrm{H},-0.50999297$ $83,-1.199892836,1.549952167 \backslash \mathrm{H}, 1.2492185252,-1.1374408974,1.3260090593 \backslash$ $\mathrm{H}, 0.4009518879,0.2056583083,-2.1257268264 \backslash \mathrm{H}, 1.2492185252,-1.1374408974$ $,-1.3260090593 \backslash \mathrm{H},-0.5099929783,-1.199892836,-1.549952167 \backslash \backslash$ Version $=\mathrm{Al} 64$ T-G03RevC.02 \State $=2-\mathrm{A}^{\prime} \backslash \mathrm{HF}=-217.716382 \backslash \mathrm{S} 2=0.753215 \backslash \mathrm{S} 2-1=0 . \backslash \mathrm{S} 2 \mathrm{~A}=0.75000$ $8 \backslash \mathrm{RMSD}=3.664 \mathrm{e}-09 \backslash \mathrm{RMSF}=2.803 \mathrm{e}-06 \backslash \mathrm{Dipole}=0.2886315,-0.5113732,0 . \backslash \mathrm{PG}=\mathrm{CS}$ [ $\mathrm{SG}(\mathrm{C} 1 \mathrm{~F} 1), \mathrm{X}(\mathrm{C} 2 \mathrm{H} 6)] \backslash \backslash @$

\section{$\left(\mathrm{CH}_{3}\right)_{2} \mathrm{CHF}$}

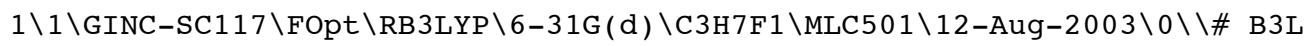
YP/6-31G* OPT=TIGHT FREQ MAXDISK $=13107200 \backslash \backslash \mathrm{Pr}-\mathrm{F}-\mathrm{Cs}-6 \mathrm{drb} 3 \backslash \backslash 0,1 \backslash \mathrm{C}, 0.3716$ $696977,0.0054355551,0 . \backslash \mathrm{H}, 1.4702007145,-0.0172750581,0 . \backslash \mathrm{F}, 0.0001158812$, $1.3598090225,0 . \backslash \mathrm{C},-0.160468291,-0.6280186387,1.2758972525 \backslash \mathrm{C},-0.1604682$ $91,-0.6280186387,-1.2758972525 \backslash \mathrm{H}, 0.2173886633,-0.0936391715,2.15327283$ $18 \backslash \mathrm{H},-1.2553374156,-0.5887127943,1.2922854481 \backslash \mathrm{H}, 0.1501275828,-1.676345$ $9393,1.3480631663 \backslash \mathrm{H}, 0.2173886633,-0.0936391715,-2.1532728318 \backslash \mathrm{H}, 0.15012$ $75828,-1.6763459393,-1.3480631663 \backslash \mathrm{H},-1.2553374156,-0.5887127943,-1.292$ $2854481 \backslash \backslash$ Version=DEC-AXP-OSF $/ 1-G 03$ RevB. 03 $\backslash$ State $=1-A^{\prime} \backslash \mathrm{HF}=-218.3780028 \backslash \mathrm{R}$ $\mathrm{MSD}=4.329 \mathrm{e}-09 \backslash \mathrm{RMSF}=1.202 \mathrm{e}-06 \backslash \mathrm{Dipole}=0.1294329,-0.6571257,0 . \backslash \mathrm{PG}=\mathrm{CS} \quad[\mathrm{SG}($ $\mathrm{C} 1 \mathrm{H} 1 \mathrm{~F} 1), \mathrm{X}(\mathrm{C} 2 \mathrm{H} 6) \mathrm{J} \backslash \backslash \mathrm{Q}$

\section{$\left(\mathrm{CH}_{3}\right)_{2} \mathrm{C} \cdot \mathrm{OCH}_{3}$}

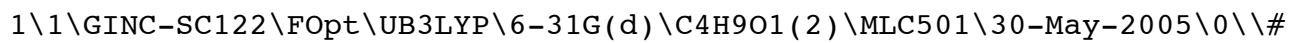
B3LYP/6-31G* OPT=TIGHT FREQ SCF=TIGHT MAXDISK=268435456 \\Me2C-OCH3-C16 dub $3 \backslash \backslash 0,2 \backslash 0,-0.7030039796,-0.6716782358,-0.1897835177 \backslash \mathrm{C},-1.9290185068$ $,-0.0056272216,0.0629020811 \backslash \mathrm{H},-2.7209049482,-0.7111873489,-0.199709211$ $1 \backslash \mathrm{H},-2.0433373771,0.8988272358,-0.5493028647 \backslash \mathrm{C}, 0.4635051287,-0.0405387$ $341,0.1920116089 \backslash \mathrm{C}, 0.5866791839,1.4452274066,0.0270692088 \backslash \mathrm{C}, 1.64921498$ $68,-0.9230334711,-0.0343010216 \backslash \mathrm{H}, 2.5248133983,-0.5421277115,0.50280940$ $67 \backslash \mathrm{H}, 1.4430432753,-1.9414220648,0.3130107591 \backslash \mathrm{H}, 1.9250492803,-0.9941472$ $481,-1.1025591666 \backslash \mathrm{H}, 1.5356655729,1.7855780111,0.4540047642 \backslash \mathrm{H}, 0.5796942$ $899,1.754374085,-1.035728955 \backslash \mathrm{H},-0.2157496842,2.0005887048,0.5267622474$ 
$\backslash \mathrm{H},-2.0265267267,0.2667743439,1.1228898987 \backslash \backslash$ Version=Al64T-G03RevC.02 $\backslash \mathrm{S}$ tate $=2-\mathrm{A} \backslash \mathrm{HF}=-233.003549 \backslash \mathrm{S} 2=0.753181 \backslash \mathrm{S} 2-1=0 . \backslash \mathrm{S} 2 \mathrm{~A}=0.750008 \backslash \mathrm{RMSD}=4.125 \mathrm{e}-0$ $9 \backslash \mathrm{RMSF}=2.515 \mathrm{e}-06 \backslash \mathrm{Dipole}=-0.1895553,0.414142,0.0571718 \backslash \mathrm{PG}=\mathrm{C} 01 \quad[\mathrm{X}(\mathrm{C} 4 \mathrm{H} 901$ ) $] \backslash \backslash$ a

\section{$\left(\mathrm{CH}_{3}\right)_{2} \mathrm{CHOCH}_{3}$}

$1 \backslash 1 \backslash$ GINC-SC15 $\backslash$ FOpt $\backslash R B 3 L Y P \backslash 6-31 G(d) \backslash C 4 H 1001 \backslash M L C 501 \backslash 26-J u n-2003 \backslash 0 \backslash \backslash \#$ B3L YP $/ 6-31 \mathrm{G} *$ OPT $=($ TIGHT, MAXCYC $=100)$ FREQ MAXDISK $=26214400 \backslash \backslash \mathrm{PrOCH} 3-\mathrm{C} 1-6 \mathrm{dub}$ $3 \backslash \backslash 0,1 \backslash 0,0.2581838741,0.937874606,0.0407424579 \backslash \mathrm{C}, 1.6365622261,1.042298$ $2793,0.3233044075 \backslash \mathrm{H}, 1.8990698734,2.1004086002,0.2355469898 \backslash \mathrm{H}, 2.2623399$ $434,0.4702401473,-0.3784687165 \backslash \mathrm{C},-0.2958120265,-0.3650962082,0.2296077$ $208 \backslash \mathrm{H}, 0.0737268667,-0.770233074,1.1884225371 \backslash \mathrm{C}, 0.0999562654,-1.3221886$ $514,-0.8997878285 \backslash \mathrm{C},-1.8070752191,-0.1766021952,0.3188323899 \backslash \mathrm{H},-2.3094$ $122994,-1.1343594496,0.4933835016 \backslash \mathrm{H},-2.0591701944,0.5065049853,1.13581$ $04054 \backslash \mathrm{H},-2.1901489088,0.2507080646,-0.6147505549 \backslash \mathrm{H},-0.3461659892,-2.31$ $07435182,-0.7410685375 \backslash \mathrm{H},-0.2515839876,-0.9340770207,-1.8625311037 \backslash \mathrm{H}, 1$ $.1853093402,-1.4555173912,-0.960633128 \backslash \mathrm{H}, 1.868776887,0.7036044613,1.34$ $6608805 \backslash \backslash$ Version $=$ DEC-AXP-OSF $/ 1-G 03$ RevB. 03 $\backslash$ State $=1-A \backslash H F=-233.660863 \backslash$ RMS $\mathrm{D}=4.763 \mathrm{e}-09 \backslash \mathrm{RMSF}=2.969 \mathrm{e}-06 \backslash \mathrm{Dipole}=0.2436283,-0.368755,0.1308948 \backslash \mathrm{PG}=\mathrm{C} 01$

$[\mathrm{X}(\mathrm{C} 4 \mathrm{H} 10 \mathrm{O} 1)] \backslash \backslash @$

\section{$\left(\mathrm{CH}_{3}\right)_{2} \mathrm{C} \cdot \mathrm{OH}$}

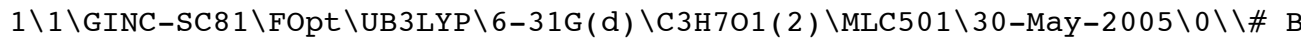
3LYP/6-31G* OPT=TIGHT FREQ SCF=TIGHT MAXDISK=402653184 GEOM=CHECK GUES $\mathrm{S}=\mathrm{READ} \backslash \backslash \mathrm{Me} 2 \mathrm{C}-\mathrm{OH}-\mathrm{C} 1-6 \mathrm{drb} 3 \backslash \backslash 0,2 \backslash 0,-0.038828155,1.3829486735,0.0595820441$ $\backslash \mathrm{H},-0.9488618124,1.6925188538,-0.0703774087 \backslash \mathrm{C}, 0.0085863548,0.027445203$ $9,-0.2213889025 \backslash \mathrm{C},-1.2365019447,-0.7637681603,0.0328520356 \backslash \mathrm{C}, 1.3628575$ $091,-0.5492057053,0.0252807929 \backslash \mathrm{H}, 1.447612788,-1.545259145,-0.422585083$ $1 \backslash \mathrm{H}, 2.1394915823,0.0927364073,-0.4056226805 \backslash \mathrm{H}, 1.5883333245,-0.65145099$ $26,1.1030145353 \backslash \mathrm{H},-1.1468846008,-1.774750999,-0.3785846976 \backslash \mathrm{H},-1.462426$ $7615,-0.8674495137,1.1113714815 \backslash \mathrm{H},-2.1162907956,-0.2967620281,-0.43433$ $60559 \backslash \backslash$ Version $=A 164 \mathrm{~T}-\mathrm{G} 03 \mathrm{RevC} .02 \backslash$ State $=2-\mathrm{A} \backslash \mathrm{HF}=-193.6979751 \backslash \mathrm{S} 2=0.753182 \backslash$ $\mathrm{S} 2-1=0 . \backslash \mathrm{S} 2 \mathrm{~A}=0.750008 \backslash \mathrm{RMSD}=2.352 \mathrm{e}-09 \backslash \mathrm{RMSF}=1.643 \mathrm{e}-06 \backslash \mathrm{Dipole}=-0.5355074$, $0.1855925,-0.0136277 \backslash \mathrm{PG}=\mathrm{C} 01 \quad[\mathrm{X}(\mathrm{C} 3 \mathrm{H} 7 \mathrm{O} 1)] \backslash \backslash @$

\section{$\left(\mathrm{CH}_{3}\right)_{2} \mathrm{CHOH}$}

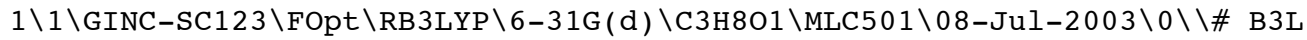
YP/6-31G* OPT FREQ GEOM=CHECK GUESS=READ MAXDISK=6553600 \PrOH-C $1-6 \mathrm{drb}$ $3 \backslash \backslash 0,1 \backslash 0,0.0371851879,1.370086144,-0.1660700928 \backslash \mathrm{H}, 0.8765112916,1.77086$ $4656,0.1089387107 \backslash \mathrm{C},-0.0017770301,0.0419150037,0.3651960081 \backslash \mathrm{H}, 0.006161$ $856,0.084188439,1.4682404055 \backslash C, 1.2045098511,-0.7746886626,-0.103439876$ $7 \backslash \mathrm{C},-1.3265199918,-0.5594800771,-0.0887419264 \backslash \mathrm{H},-1.4584660428,-1.56636$ $33148,0.3221508884 \backslash \mathrm{H},-2.1609479598,0.0659837628,0.2435658552 \backslash \mathrm{H},-1.3593$ $617406,-0.620871502,-1.18243542 \backslash \mathrm{H}, 1.1769644107,-1.7929296921,0.3021373$ $049 \backslash \mathrm{H}, 1.2194243672,-0.8349148071,-1.1975802921 \backslash \mathrm{H}, 2.1449553389,-0.31312$ $4277,0.2254580602 \backslash \backslash$ Version=DEC-AXP-OSF $/ 1-G 03$ RevB.03 $\backslash$ State $=1-A \backslash \mathrm{HF}=-194$. $3533176 \backslash \mathrm{RMSD}=7.543 e-10 \backslash \mathrm{RMSF}=2.547 \mathrm{e}-06 \backslash \mathrm{Dipole}=0.4565079,-0.2535501,0.32$ $14917 \backslash \mathrm{PG}=\mathrm{C} 01 \quad[\mathrm{X}(\mathrm{C} 3 \mathrm{H} 8 \mathrm{O} 1)] \backslash \backslash @$

\section{$\left(\mathrm{CH}_{3}\right)_{2} \mathrm{C} \cdot \mathrm{SCH}_{3}$}

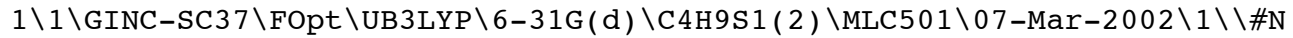
UB3LYP $/ 6-31 \mathrm{G} *$ FOPT $=(Z-M A T R I X) \quad S C F=T I G H T$ FREQ MAXDISK $=3932160000$ GEOM=C $\mathrm{HECK} \backslash \backslash \mathrm{CH} 3-\mathrm{S}-{ }^{*} \mathrm{C}(\mathrm{CH} 3) 2$ in $\mathrm{C} 1$ symm $\backslash \backslash 0,2 \backslash \mathrm{S} \backslash \mathrm{C}, 1, \mathrm{~B} 1 \backslash \mathrm{C}, 2, \mathrm{~B} 2,1, \mathrm{~A} 1 \backslash \mathrm{C}, 2, \mathrm{~B} 3,1, \mathrm{~A} 2$, 3 , D1 , $0 \backslash \mathrm{C}, 1, \mathrm{~B} 4,2, \mathrm{~A} 3,3, \mathrm{D} 2,0 \backslash \mathrm{H}, 5, \mathrm{~B} 5,1, \mathrm{~A} 4,2, \mathrm{D} 3,0 \backslash \mathrm{H}, 5, \mathrm{~B} 6,1, \mathrm{~A} 5,6, \mathrm{D} 4,0 \backslash \mathrm{H}, 5, \mathrm{~B} 7$ $, 1, \mathrm{~A} 6,6, \mathrm{D} 5,0 \backslash \mathrm{H}, 3, \mathrm{~B} 8,2, \mathrm{~A} 7,1, \mathrm{D} 6,0 \backslash \mathrm{H}, 3, \mathrm{~B} 9,2, \mathrm{~A} 8,1, \mathrm{D} 7,0 \backslash \mathrm{H}, 3, \mathrm{~B} 10,2, \mathrm{~A} 9,1, \mathrm{D} 8,0$ $\backslash \mathrm{H}, 4, \mathrm{~B} 11,2, \mathrm{~A} 10,1, \mathrm{D} 9,0 \backslash \mathrm{H}, 4, \mathrm{~B} 12,2, \mathrm{~A} 11,1, \mathrm{D} 10,0 \backslash \mathrm{H}, 4, \mathrm{~B} 13,2, \mathrm{~A} 12,1, \mathrm{D} 11,0 \backslash \backslash \mathrm{B} 1=$ $1.76040029 \backslash \mathrm{B} 2=1.50116469 \backslash \mathrm{B} 3=1.49930006 \backslash \mathrm{B} 4=1.82899497 \backslash \mathrm{B} 5=1.09299456 \backslash \mathrm{B} 6=$ $1.09335041 \backslash \mathrm{B} 7=1.09272043 \backslash \mathrm{A} 1=114.32311171 \backslash \mathrm{A} 2=121.81593508 \backslash \mathrm{A} 3=104.030368$ $33 \backslash A 4=106.04568798 \backslash A 5=110.89519235 \backslash A 6=111.94271848 \backslash D 1=-154.0787177 \backslash D 2=$ $-169.04426525 \backslash D 3=-186.43130151 \backslash D 4=-118.19677541 \backslash D 5=118.46758739 \backslash B 8=1.0$ $9556304 \backslash \mathrm{B} 9=1.10437127 \backslash \mathrm{B} 10=1.09829993 \backslash \mathrm{B} 11=1.10516989 \backslash \mathrm{B} 12=1.09504758 \backslash \mathrm{B} 13$ $=1.0974191 \backslash \mathrm{A} 7=111.97869626 \backslash \mathrm{A} 8=112.51774977 \backslash \mathrm{A} 9=110.25141341 \backslash \mathrm{A} 10=112.165$ 
$31975 \backslash A 11=113.04786072 \backslash A 12=109.97870399 \backslash D 6=34.59578555 \backslash D 7=-86.38557568$ $\backslash \mathrm{D} 8=154.77050823 \backslash \mathrm{D} 9=77.19465282 \backslash \mathrm{D} 10=-43.91014241 \backslash \mathrm{D} 11=195.9695628 \backslash \backslash$ Vers ion=DEC-AXP-OSF $/ 1-\mathrm{G} 98 \mathrm{RevA} .9 \backslash \mathrm{HF}=-555.9879304 \backslash \mathrm{S} 2=0.754442 \backslash \mathrm{S} 2-1=0 . \backslash \mathrm{S} 2 \mathrm{~A}=0$. $750015 \backslash \mathrm{RMSD}=4.175 \mathrm{e}-09 \backslash \mathrm{RMSF}=1.747 \mathrm{e}-05 \backslash \mathrm{Dipole}=-0.5112718,-0.0022178,0.22$ $33791 \backslash \mathrm{PG}=\mathrm{C} 01 \quad[\mathrm{X}(\mathrm{C} 4 \mathrm{H} 9 \mathrm{~S} 1)] \backslash \backslash @$

\section{$\left(\mathrm{CH}_{3}\right)_{2} \mathrm{CHSCH}_{3}$}

$1 \backslash 1 \backslash$ GINC-SC8 $1 \backslash$ FOpt $\backslash R B 3 L Y P \backslash 6-31 G(d) \backslash C 4 H 10 S 1 \backslash M L C 501 \backslash 30-M a y-2005 \backslash 0 \backslash \backslash \#$ B3L YP $/ 6-31 \mathrm{G} *$ OPT FREQ=NORAMAN MAXDISK $=268435456 \backslash \backslash \mathrm{Me} 2 \mathrm{CH}-\mathrm{SCH} 3-\mathrm{C} 1-6 \mathrm{drb} 3 \backslash \backslash 0,1$ $\backslash S, 0.7336720854,-0.7252505216,-0.3768356797 \backslash C,-0.7123226234,0.13750487$ $5,0.3938031119 \backslash \mathrm{C},-1.9396155541,-0.7564106069,0.1892568743 \backslash \mathrm{C},-0.9328839$ $072,1.5431085302,-0.1710472726 \backslash \mathrm{C}, 2.1247273994,0.2794397435,0.250674603$ $2 \backslash \mathrm{H}, 3.0422441345,-0.214108602,-0.0808328808 \backslash \mathrm{H}, 2.1239925956,0.315397068$ $3,1.3449123881 \backslash \mathrm{H}, 2.1129623379,1.2967419209,-0.1507420476 \backslash \mathrm{H},-1.79668237$ $64,-1.7429766067,0.6410347149 \backslash \mathrm{H},-2.1506327666,-0.8956533651,-0.8775986$ $903 \backslash \mathrm{H},-2.8214636085,-0.2922841131,0.6466743783 \backslash \mathrm{H},-1.1466511644,1.50055$ $65197,-1.2446863426 \backslash \mathrm{H},-0.0594830002,2.1867721431,-0.0241614988 \backslash \mathrm{H},-1.78$ $4280776,2.0220224982,0.3303915884 \backslash \mathrm{H},-0.4981906303,0.2056856309,1.46825$ $53644 \backslash \backslash$ Version=Al64T-G03RevC.02 $\backslash$ State $=1-\mathrm{A} \backslash \mathrm{HF}=-556.6427746 \backslash \mathrm{RMSD}=8.029 \mathrm{e}-$ $09 \backslash \mathrm{RMSF}=1.104 \mathrm{e}-05 \backslash \mathrm{Dipole}=-0.0743159,0.5332441,0.4079326 \backslash \mathrm{PG}=\mathrm{C} 01 \quad[\mathrm{X}(\mathrm{C} 4 \mathrm{H} 1$ $0 \mathrm{~S} 1)] \backslash \backslash \mathrm{Q}$

\section{$\cdot \mathrm{P}\left(\mathrm{CH}_{3}\right)_{4}$}

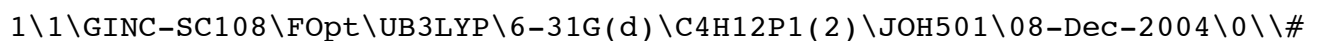
B3LYP/6-31G* OPT FREQ MAXDISK $=65536000 \backslash \backslash$ pch34_5 opt freq b3lyp $\backslash \backslash 0,2 \backslash P$ $, 0 ., 0.0 .3115948418 \backslash \mathrm{C}, 1.9779003667,0 ., 0.6232678829 \backslash \mathrm{C},-1.9779003667,0$. , $0.6232678829 \backslash \mathrm{C}, 0 .,-1.4676715436,-0.8330835307 \backslash \mathrm{C}, 0,1.4676715436,-0.833$ $0835307 \backslash \mathrm{H}, 2.5484816755,0 .,-0.3158410104 \backslash \mathrm{H},-2.5484816755,0 .,-0.31584101$ $04 \backslash \mathrm{H}, 2.2304646838,-0.8897497538,1.2084273618 \backslash \mathrm{H}, 2.2304646838,0.88974975$ $38,1.2084273618 \backslash \mathrm{H},-2.2304646838,0.8897497538,1.2084273618 \backslash \mathrm{H},-2.2304646$ $838,-0.8897497538,1.2084273618 \backslash \mathrm{H}, 0 .,-2.3908292027,-0.2426842864 \backslash \mathrm{H}, 0 ., 2$ $.3908292027,-0.2426842864 \backslash \mathrm{H},-0.8908674176,-1.4713549552,-1.4681984268 \backslash$ $\mathrm{H}, 0.8908674176,-1.4713549552,-1.4681984268 \backslash \mathrm{H}, 0.8908674176,1.4713549552$ $,-1.4681984268 \backslash \mathrm{H},-0.8908674176,1.4713549552,-1.4681984268 \backslash \backslash$ Version=DEC $-\mathrm{AXP}-\mathrm{OSF} / 1-\mathrm{G} 03 \mathrm{RevB} .03 \backslash \mathrm{State}=2-\mathrm{A} 1 \backslash \mathrm{HF}=-500.9355065 \backslash \mathrm{S} 2=0.755056 \backslash \mathrm{S} 2-1=0 . \backslash \mathrm{S}$ $2 \mathrm{~A}=0.750019 \backslash \mathrm{RMSD}=5.703 \mathrm{e}-09 \backslash \mathrm{RMSF}=2.969 \mathrm{e}-05 \backslash \mathrm{Dipole}=0,, 0 .,-0.384381 \backslash \mathrm{PG}=\mathrm{C} 0$ $2 \mathrm{~V}\left[\mathrm{C} 2(\mathrm{P} 1), \mathrm{SGV}(\mathrm{C} 2 \mathrm{H} 2), \mathrm{SGV}^{\prime}(\mathrm{C} 2 \mathrm{H} 2), \mathrm{X}(\mathrm{H} 8)\right] \backslash \backslash \mathrm{Q}$

\section{$\mathrm{HP}\left(\mathrm{CH}_{3}\right)_{3} \cdot \mathrm{CH}_{2}$}

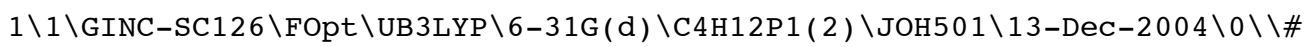
B3LYP/6-31G* OPT FREQ MAXDISK=65536000\\rC_1b3_6db3 opt freq b3lyp \\0 $, 2 \backslash \mathrm{P},-0.0341487167,0 .,-0.2901707603 \backslash \mathrm{C},-1.93 \overline{8} 626 \overline{8} 925,0 .,-0.4893126857 \backslash \mathrm{C}$ $, 1.911835811,0 .,-0.404851802 \backslash \mathrm{H}, 2.4332428782,0 ., 0.5617860142 \backslash \mathrm{H},-2.38421$ $07761,0 .,-1.4867484424 \backslash \mathrm{H},-2.6583826073,0 ., 0.3348507006 \backslash \mathrm{H}, 2.236619252,0$ $.8848041797,-0.9704941126 \backslash \mathrm{H}, 2.236619252,-0.8848041797,-0.9704941126 \backslash \mathrm{C}$, $-0.0629231142,1.5724209013,0.7167147295 \backslash \mathrm{C},-0.0629231142,-1.5724209013$, $0.7167147295 \backslash \mathrm{H},-0.9581438914,1.6176623937,1.3416516157 \backslash \mathrm{H},-0.9581438914$ $,-1.6176623937,1.3416516157 \backslash \mathrm{H},-0.0961496056,2.4170895175,0.0198389494 \backslash$ $\mathrm{H},-0.0961496056,-2.4170895175,0.0198389494 \backslash \mathrm{H}, 0.8378616486,1.6645197064$ $, 1.3295691836 \backslash \mathrm{H}, 0.8378616486,-1.6645197064,1.3295691836 \backslash \mathrm{H},-0.002969692$ $2,0 .,-1.7340479679 \backslash \backslash$ Version=DEC-AXP-OSF $/ 1-$ G03RevB.03 $\backslash$ State $=2-A^{\prime \prime} \backslash H F=-50$ $0.8663257 \backslash \mathrm{S} 2=0.754235 \backslash \mathrm{S} 2-1=0 . \backslash \mathrm{S} 2 \mathrm{~A}=0.75001 \backslash \mathrm{RMSD}=7.033 \mathrm{e}-09 \backslash \mathrm{RMSF}=1.009 \mathrm{e}-0$ $5 \backslash$ Dipole $=-0.0413117,0 ., 0.3134812 \backslash \mathrm{PG}=\mathrm{CS}[\mathrm{SG}(\mathrm{C} 2 \mathrm{H} 4 \mathrm{P} 1), \mathrm{X}(\mathrm{C} 2 \mathrm{H} 8)] \backslash \backslash @$

\section{$\cdot \mathrm{P}\left(\mathrm{CH}_{3}\right)_{3} \mathrm{CH}_{2} \mathrm{CH}_{3}$}

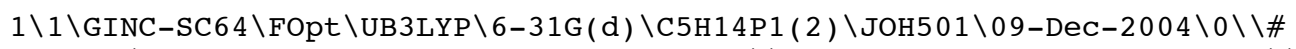
B3LYP/6-31G* OPT FREQ MAXDISK=134217728\\rC_2a1_6db3 opt freq b31yp $\backslash \backslash 0$ $, 2 \backslash \mathrm{C}, 2.3068995934,-0.2009714538,-0.40299527 \overline{2} 6 \backslash \mathrm{P}, 0.3484801207,-0.171152$ $0699,-0.1027951665 \backslash C,-1.6798467223,-0.1555837451,-0.4073472228 \backslash C, 0.322$ $9601617,-1.6593735239,1.0179020731 \backslash \mathrm{C}, 0.3828518248,1.2678461816,1.08086$ $78073 \backslash \mathrm{H}, 1.1866301829,1.144477718,1.8137473686 \backslash \mathrm{H},-0.5680574402,1.369721$ $0621,1.6119190364 \backslash \mathrm{H}, 0.5693118321,2.1956371556,0.5295217195 \backslash \mathrm{H}, 1.1670624$ $067,-1.6388168623,1.7144900426 \backslash \mathrm{H}, 0.4023343705,-2.5714583701,0.41575471$ 
$28 \backslash \mathrm{H},-0.6078398413,-1.7097377107,1.5901011975 \backslash \mathrm{H}, 2.8747458795,-0.218942$ $3957,0.5379277228 \backslash \mathrm{H}, 2.5536240981,-1.0881949569,-0.9949843218 \backslash \mathrm{H}, 2.58176$ $58317,0.6888882855,-0.9782861487 \backslash \mathrm{H},-2.1672914522,-0.1477856257,0.57769$ $23647 \backslash \mathrm{C},-2.109150725,1.023300543,-1.269451853 \backslash \mathrm{H},-1.8993765756,-1.11178$ $5534,-0.8962457423 \backslash \mathrm{H},-3.1844620371,0.9966571017,-1.4908727984 \backslash \mathrm{H},-1.903$ $6147725,1.9812164158,-0.7754029123 \backslash \mathrm{H},-1.574319089,1.0260967548,-2.2272$ $879358 \backslash \backslash$ Version=DEC-AXP-OSF $/ 1-G 03$ RevB.03 $\backslash$ State $=2-A \backslash H F=-540.2485175 \backslash \mathrm{S} 2=$ $0.755524 \backslash \mathrm{S} 2-1=0 . \backslash \mathrm{S} 2 \mathrm{~A}=0.750021 \backslash \mathrm{RMSD}=5.563 \mathrm{e}-09 \backslash \mathrm{RMSF}=7.726 \mathrm{e}-06 \backslash \mathrm{Dipole}=0.0$ $205405,-0.0291615,0.3835461 \backslash P G=C 01 \quad[X(C 5 H 14 P 1)] \backslash \backslash @$

\section{$\mathrm{CH}_{3} \mathrm{P}\left(\mathrm{CH}_{3}\right)_{3} \cdot \mathrm{CH}_{2}$}

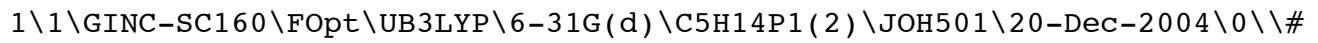
B3LYP/6-31G* OPT FREQ SCF=QC MAXDISK=268435456\\rC_2b3_6db5 opt freq b3lyp $\backslash \backslash 0,2 \backslash P, 0.0051595924,0.0007144372,-0.005842810 \overline{6} \backslash \mathrm{C},-1.9538533464,-$ $0.0027092228,0.0249226571 \backslash \mathrm{C}, 1.9542247665,0.0224921906,-0.0304124869 \backslash \mathrm{C}$, $0.0374504737,1.8364094364,-0.4003338005 \backslash C, 0.0466188501,-1.2708050665,-$ $1.3811109144 \backslash \mathrm{H},-2.3487844019,-1.0038375836,0.2456615151 \backslash \mathrm{H},-2.366232808$ $3,0.3137665695,-0.9431947864 \backslash \mathrm{H},-2.3381960487,0.6845102339,0.7915192994$ $\backslash \mathrm{H}, 0.5530194229,2.3589788524,0.4125595006 \backslash \mathrm{H}, 0.6598635629,-2.1201302748$ $,-1.0641581184 \backslash \mathrm{H}, 0.6256909638,1.9907200063,-1.310298883 \backslash \mathrm{H},-0.960654503$ $7,2.2599487441,-0.5268663962 \backslash \mathrm{H},-0.9485784791,-1.6224577996,-1.66253505$ $76 \backslash \mathrm{H}, 0.5388683245,-0.8220172364,-2.2498544541 \backslash \mathrm{C}, 0.0854359602,-0.585138$ $7055,1.7761444639 \backslash \mathrm{H}, 2.5535915683,0.9261792071,0.1011623401 \backslash \mathrm{H}, 2.5472904$ $978,-0.8961766089,-0.0454024895 \backslash \mathrm{H}, 0.556885363,0.2021175732,2.373943749$ $4 \backslash \mathrm{H},-0.8990232688,-0.8163161221,2.1923355184 \backslash \mathrm{H}, 0.7296056969,-1.4674939$ $12,1.8375109049 \backslash \backslash$ Version=DEC-AXP-OSF $/ 1-G 03$ RevB .03 $\backslash$ State $=2-A \backslash H F=-540.18$ $41944 \backslash \mathrm{S} 2=0.754424 \backslash \mathrm{S} 2-1=0 . \backslash \mathrm{S} 2 \mathrm{~A}=0.750011 \backslash \mathrm{RMSD}=0.000 \mathrm{e}+00 \backslash \mathrm{RMSF}=2.199 \mathrm{e}-05 \backslash \mathrm{D}$ ipole $=0.0530042,0.0000135,0.0280289 \backslash \mathrm{PG}=\mathrm{C} 01 \quad[\mathrm{X}(\mathrm{C} 5 \mathrm{H} 14 \mathrm{P} 1)] \backslash \backslash @$

\section{$\cdot \mathrm{P}\left(\mathrm{CH}_{3}\right)_{3} \mathrm{CH}_{2} \mathrm{C}\left(\mathrm{CH}_{3}\right)_{3}$}

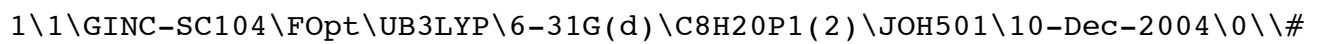
B3LYP/6-31G* OPT FREQ MAXDISK=268435456\\rC_3a1_6db3 opt freq b3lyp $\backslash \backslash$ $0,2 \backslash \mathrm{C}, 3.0363156915,-0.5477088998,0.214073739 \overline{4} \backslash \mathrm{P}, \overline{1} .0817834847,-0.510471$ $0899,0.4524758397 \backslash C,-1.0052577469,-0.4170877692,0.0943272894 \backslash C, 0.95684$ $13451,-2.215527464,1.1946027645 \backslash \mathrm{C}, 1.1169803722,0.5797908826,1.96462412$ $41 \backslash \mathrm{H}, 1.8777984238,0.2294966421,2.6701054308 \backslash \mathrm{H}, 0.1474246531,0.595514401$ $7,2.4690727715 \backslash \mathrm{H}, 1.368971179,1.6056052781,1.6764905349 \backslash \mathrm{H}, 1.7821448051$, $-2.4000365369,1.8895427252 \backslash \mathrm{H}, 1.0042232828,-2.9676827311,0.3992531593 \backslash \mathrm{H}$ $, 0.0097226392,-2.3427648949,1.7266555607 \backslash \mathrm{H}, 3.5691324082,-0.8216141114$, $1.1355191824 \backslash \mathrm{H}, 3.2788879554,-1.2698079953,-0.5724950018 \backslash \mathrm{H}, 3.3664197935$ $, 0.4434148764,-0.1139799184 \backslash \mathrm{H},-1.46846841,-0.2916929157,1.082960399 \backslash \mathrm{C}$, $-1.4886161181,0.6481533308,-0.899594182 \backslash \mathrm{H},-1.2373837298,-1.4234397353$, $-0.2756246959 \backslash C,-3.0325269493,0.5884116932,-0.9705399379 \backslash C,-1.07094884$ $93,2.0607685035,-0.4464990773 \backslash \mathrm{C},-0.9172811859,0.3709358217,-2.30383005$ $05 \backslash \mathrm{H},-1.4833793717,2.8241682337,-1.1180267143 \backslash \mathrm{H},-1.4329296183,2.277591$ $7496,0.566393495 \backslash \mathrm{H}, 0.0194199785,2.1737302806,-0.4487397361 \backslash \mathrm{H},-3.422328$ $5335,1.3222566589,-1.6889531971 \backslash \mathrm{H},-3.3755079052,-0.404437037,-1.285958$ $3219 \backslash \mathrm{H},-3.4832205938,0.8034190057,0.0061777015 \backslash \mathrm{H},-1.2728289711,1.11693$ $86938,-3.0257940896 \backslash \mathrm{H}, 0.1778379265,0.3978703042,-2.2964500937 \backslash \mathrm{H},-1.225$ $7275387,-0.6178804117,-2.6662748058 \backslash \backslash$ Version=DEC-AXP-OSF / 1-G03RevB.03\ State $=2-A \backslash H F=-658.1872164 \backslash S 2=0.756465 \backslash S 2-1=0 . \backslash S 2 A=0.750025 \backslash R M S D=9.242 e$ $-09 \backslash \mathrm{RMSF}=4.633 e-06 \backslash \mathrm{Dipole}=0.0940856,-0.1418245,0.3866461 \backslash \mathrm{PG}=\mathrm{C} 01 \quad[\mathrm{X}(\mathrm{C} 8 \mathrm{H} 20 \mathrm{P} 1)] \backslash \backslash @$

\section{$\mathrm{CH}_{3} \mathrm{P}\left(\mathrm{CH}_{3}\right)_{3} \mathrm{CH}_{2} \cdot \mathrm{C}\left(\mathrm{CH}_{3}\right)_{2}$}

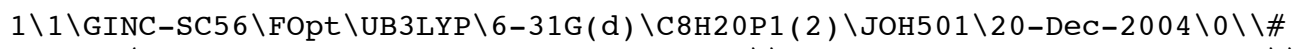
B3LYP/6-31G* OPT FREQ MAXDISK=268435456\\rC_3b7_6db3 opt freq b31yp $\backslash \backslash 0$ $, 2 \backslash C, 2.8450999998,-0.0025502404,-0.78957 \backslash P, \overline{1} .06 \overline{6} 4900001,0.0011699099,0$ $.0126 \backslash C,-0.8299700001,-0.0013199299,0.96145 \backslash C, 1.8559299972,-0.03306015$ $68,1.72204 \backslash \mathrm{C}, 0.6303001379,1.6322099467,-0.79248 \backslash \mathrm{H}, 1.4096701682,1.99096$ $98809,-1.46796 \backslash \mathrm{H}, 0.4612902005,2.373119961,-0.00495 \backslash \mathrm{H},-0.3089298727,1.5$ $072400261,-1.33603 \backslash \mathrm{H}, 2.5653900673,0.7964597833,1.79737 \backslash \mathrm{H}, 2.4269199186$, $-0.962940205,1.81225 \backslash \mathrm{H}, 1.1305700023,0.0270699045,2.5341 \backslash \mathrm{H}, 3.4240000744$ $, 0.8807797107,-0.49008 \backslash \mathrm{H}, 3.4158699245,-0.8940102886,-0.49827 \backslash \mathrm{H}, 2.77227$ 
$00002,0.0029397658,-1.88495 \backslash \mathrm{H},-0.753129925,0.8874300636,1.60613 \backslash \mathrm{C},-2.0$ $7122,-0.000419825,0.18754 \backslash \mathrm{H},-0.7523200754,-0.8924099364,1.60281 \backslash \mathrm{C}, 0.60$ $29898653,-1.5948700509,-0.84766 \backslash \mathrm{C},-2.7869298924,1.2736002355,-0.16264 \backslash$ $\mathrm{C},-2.7886901076,-1.2734497644,-0.16255 \backslash \mathrm{H},-2.9561498827,1.3883502498,-1$ $.24741 \backslash \mathrm{H},-3.7921198895,1.3083303204,0.29421 \backslash \mathrm{H},-2.2457198173,2.16247018$ $97,0.17966 \backslash \mathrm{H},-2.963280117,-1.3851697496,-1.24672 \backslash \mathrm{H},-2.2454701828,-2.16$ $31298103,0.1743 \backslash \mathrm{H},-3.7914701106,-1.3096096797,0.29948 \backslash \mathrm{H}, 0.4005098011,-$ $2.3543000338,-0.08625 \backslash \mathrm{H},-0.3237001207,-1.4283799727,-1.40178 \backslash \mathrm{H}, 1.38349$ $98349,-1.9536601169,-1.52176 \backslash \backslash$ Version=DEC-AXP-OSF / 1-G03RevB.03\State=2 $-\mathrm{A} \backslash \mathrm{HF}=-658.1400837 \backslash \mathrm{S} 2=0.75628 \backslash \mathrm{S} 2-1=0 . \backslash \mathrm{S} 2 \mathrm{~A}=0.750027 \backslash \mathrm{RMSD}=7.936 \mathrm{e}-09 \backslash \mathrm{RMSF}$ $=1.109 e-05 \backslash \mathrm{Dipole}=0.4352227,-0.0005591,-0.0234079 \backslash \mathrm{PG}=\mathrm{C} 01 \quad[\mathrm{X}(\mathrm{C} 8 \mathrm{H} 20 \mathrm{P} 1)] \backslash \backslash @$

\section{$\cdot \mathrm{C}\left(\mathrm{CH}_{3}\right)_{3}$}

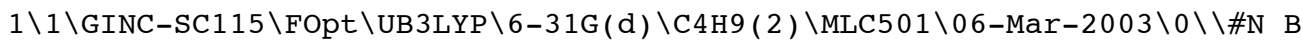
3LYP/6-31G(D) OPT=TIGHT FREQ MAXDISK=26214400\\Bu_rad Cs $\backslash \backslash 0,2 \backslash \mathrm{H},-1.134$ $0543532,1.7616707466,0 . \backslash C,-1.3291518262,0.6720547 \overline{3} 43,0 . \backslash C,-0.063598760$ $4,-0.1288255571,0 . \backslash \mathrm{C}, 0.6742610965,-0.3163631938,1.2896997828 \backslash \mathrm{C}, 0.67426$ $10964,-0.3163631938,-1.2896997829 \backslash \mathrm{H},-1.9400616179,0.4675010949,0.88858$ $77545 \backslash \mathrm{H},-1.940061618,0.4675010949,-0.8885877544 \backslash \mathrm{H}, 1.2835911529,0.56875$ $54926,1.555440113 \backslash \mathrm{H},-0.0116451147,-0.4829080616,2.1301907067 \backslash \mathrm{H}, 1.36782$ $79377,-1.165692268,1.2415727567 \backslash \mathrm{H}, 1.2835911528,0.5687554926,-1.5554401$ $131 \backslash \mathrm{H},-0.0116451148,-0.4829080615,-2.1301907067 \backslash \mathrm{H}, 1.3678279377,-1.1656$ $92268,-1.2415727567 \backslash \backslash$ Version=DEC-AXP-OSF $/ 1-G 98 R e v A .11 .3 \backslash$ State $=2-A^{\prime} \backslash \mathrm{HF}=$ $-157.7983276 \backslash \mathrm{S} 2=0.75402 \backslash \mathrm{S} 2-1=0 . \backslash \mathrm{S} 2 \mathrm{~A}=0.750012 \backslash \mathrm{RMSD}=4.543 \mathrm{e}-09 \backslash \mathrm{RMSF}=2.179$ e-06\Dipole $=0.0334859,0.0680061,0 . \backslash P G=C S \quad[S G(C 2 H 1), x(C 2 H 8)] \backslash \backslash @$

\section{$\mathrm{H}_{2} \mathrm{C}=\mathrm{P}\left(\mathrm{CH}_{3}\right)_{3}$}

$1 \backslash 1 \backslash G I N C-L C 60 \backslash F O p t \backslash R B 3 L Y P \backslash 6-31 G(d) \backslash C 4 H 11 P 1 \backslash J O H 501 \backslash 21-A p r-2005 \backslash 0 \backslash \backslash \#$ B3L

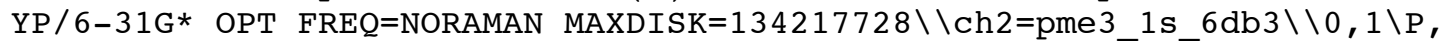
$-0.1613276512,0.0274846539,0 . \backslash \mathrm{C},-1.8428902637,0.026831 \overline{3} 00 \overline{2}, 0 . \backslash \mathrm{C}, 0.9006$ $209705,1.575529116,0 . \backslash \mathrm{H}, 1.9772376085,1.3623139231,0 . \backslash \mathrm{C}, 0.4860054985,-0$ $.8508485125,-1.4773662373 \backslash \mathrm{C}, 0.4860054985,-0.8508485125,1.4773662373 \backslash \mathrm{H}$, $-2.3424617681,0.3181398319,-0.9210323672 \backslash \mathrm{H},-2.3424617681,0.3181398319$, $0.9210323672 \backslash \mathrm{H}, 0.6576027576,2.1730313327,-0.8853611887 \backslash \mathrm{H}, 0.6576027576$, $2.1730313327,0.8853611887 \backslash \mathrm{H}, 1.5802067765,-0.8390616002,-1.5032959248 \backslash \mathrm{H}$ $, 1.5802067765,-0.8390616002,1.5032959248 \backslash \mathrm{H}, 0.1110608083,-0.3591921229$, $-2.3810328615 \backslash \mathrm{H}, 0.1110608083,-0.3591921229,2.3810328615 \backslash \mathrm{H}, 0.1257048936$ $,-1.882199481,-1.4714770407 \backslash \mathrm{H}, 0.1257048936,-1.882199481,1.4714770407 \backslash \backslash$ Version=IA32L-G03RevC.02 $\backslash$ State $=1-A^{\prime} \backslash$ HF $=-500.3524413 \backslash$ RMSD $=7.394 e-09 \backslash$ RMS $\mathrm{F}=1.097 \mathrm{e}-05 \backslash \mathrm{Dipole}=1.144614,0.0778383,0 . \backslash \mathrm{PG}=\mathrm{CS} \quad[\mathrm{SG}(\mathrm{C} 2 \mathrm{H} 1 \mathrm{P} 1), \mathrm{X}(\mathrm{C} 2 \mathrm{H} 10)] \backslash \backslash$ @

\section{$\mathrm{CH}_{3} \mathrm{CH}_{2} \mathrm{P} \cdot\left(\mathrm{CH}_{3}\right)_{3}$}

$1 \backslash 1 \backslash G I N C-S C 64 \backslash F O p t \backslash U B 3 L Y P \backslash 6-31 G$ (d) \C5H14P1 ( 2$) \backslash J O H 501 \backslash 21-A p r-2005 \backslash 0 \backslash \backslash \#$

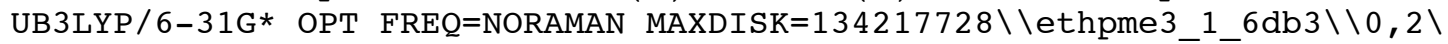
$\mathrm{C},-1.7292451657,-0.0387074223,0.141890708 \backslash \mathrm{P}, 0.3164918561, \overline{0} . \overline{0} 766319992$, $0.235154556 \backslash \mathrm{C}, 0.3707042311,1.9350186343,0.1136860461 \backslash \mathrm{C}, 2.1981393237,-0$ $.1238363662,0.822599856 \backslash \mathrm{C}, 0.6267172394,-0.4857591521,-1.5142950193 \backslash \mathrm{H}, 0$ $.7802969812,-1.5700259088,-1.5322961382 \backslash \mathrm{H}, 1.5273247469,-0.0114991111,-$ $1.9172432305 \backslash \mathrm{H},-0.2199236683,-0.2485197939,-2.1650973218 \backslash \mathrm{H}, 2.436722472$ $6,-1.1916054524,0.8591970155 \backslash \mathrm{H}, 2.292162644,0.2886004644,1.8323437238 \backslash \mathrm{H}$ , 2.9034088906,0.3863938956,0.1514953302\H,1.3114955003,2.2701632555,-0 $.3343901992 \backslash \mathrm{H},-0.4623675282,2.3186227595,-0.4822625671 \backslash \mathrm{H}, 0.2996525493$, $2.3689079817,1.1174126217 \backslash \mathrm{C},-2.2108826689,-1.4827894015,0.1427907367 \backslash \mathrm{H}$ $,-3.3070094841,-1.547233513,0.1622634218 \backslash \mathrm{H},-1.8702711769,-2.0229556928$ $,-0.74947332 \backslash \mathrm{H},-1.8322746223,-2.0236511129,1.0189325695 \backslash \mathrm{H},-2.083186214$ $2,0.5032686613,1.0265111093 \backslash \mathrm{H},-2.0560066904,0.5064958268,-0.7547453196$ $\backslash \backslash$ Version=Al64 T-G03RevC.02 \State=2-A \HF=-540.2485175 $\backslash \mathrm{S} 2=0.755522 \backslash \mathrm{S} 2-1=$ $0 . \backslash S 2 A=0.750021 \backslash R M S D=7.329 e-09 \backslash R M S F=1.261 e-05 \backslash D i p o l e=0.078186,0.232676$ 


\section{$9,-0.2967816 \backslash \mathrm{PG}=\mathrm{C} 01 \quad[\mathrm{X}(\mathrm{C} 5 \mathrm{H} 14 \mathrm{P} 1)] \backslash \backslash @$}

\section{$\left(\mathrm{CH}_{3}\right)_{3} \mathrm{C}-\mathrm{C}\left(\mathrm{CH}_{3}\right)_{3}$}

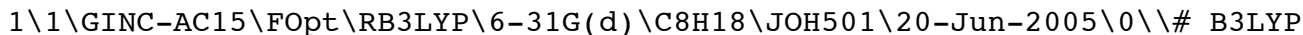
/6-31G* OPT FREQ=NORAMAN MAXDISK=268435456\\me3ccme3_1 opt freq b3lyp \} $\backslash 0,1 \backslash \mathrm{C},-0.794924793,0.0246734048,-0.00009743 \backslash \mathrm{C},-1.33 \overline{3} 503218,0.86857691$ $11,1.1785825163 \backslash \mathrm{C},-1.3372061519,0.6494140653,-1.3067866826 \backslash \mathrm{H},-0.940679$ $8209,0.5321472656,2.1447899969 \backslash \mathrm{H},-1.1447649429,0.013016848,-2.17677327$ $71 \backslash \mathrm{H},-1.0926369789,1.9308941347,1.0691027287 \backslash \mathrm{H},-0.9038626849,1.6355320$ $104,-1.5076806298 \backslash \mathrm{H},-2.4261252081,0.7864209348,1.2261495045 \backslash \mathrm{H},-2.42368$ $96212,0.779988638,-1.2333492279 \backslash \mathrm{C}, 0.7952567663,-0.0247040751,0.0002671$ $219 \backslash \mathrm{C},-1.4027118771,-1.3912041585,0.1262637963 \backslash \mathrm{C}, 1.3402262492,-0.61268$ $64385,-1.3218959159 \backslash \mathrm{C}, 1.4025485554,1.3874163262,0.1678217217 \backslash \mathrm{C}, 1.33060$ $16098,-0.90129307,1.1558305768 \backslash \mathrm{H},-1.0243560391,-2.0777414663,-0.639565$ $2253 \backslash \mathrm{H},-1.2052042078,-1.8381961212,1.1059923309 \backslash \mathrm{H},-2.4916952864,-1.340$ $4770036,0.0062225906 \backslash \mathrm{H}, 2.4259762187,-0.7496788062,-1.24971105 \backslash \mathrm{H}, 0.9048$ $273327,-1.5911468418,-1.5531553704 \backslash \mathrm{H}, 1.1528861072,0.0485636143,-2.1741$ $232038 \backslash \mathrm{H}, 2.4235764265,-0.8250741225,1.2044501857 \backslash \mathrm{H}, 0.9402458521,-0.587$ $6208807,2.130491371 \backslash \mathrm{H}, 1.0844949617,-1.9593245209,1.0189126916 \backslash \mathrm{H}, 2.4911$ $819354,1.341653955,0.0424771554 \backslash \mathrm{H}, 1.0201688915,2.0957763635,-0.5753986$ $008 \backslash \mathrm{H}, 1.2079342196,1.8041082078,1.1612538023 \backslash \backslash$ Version=IA64L-G03RevC 02 $\backslash$ State $=1-\mathrm{A} \backslash \mathrm{HF}=-315.7013964 \backslash \mathrm{RMSD}=9.675 \mathrm{e}-09 \backslash \mathrm{RMSF}=1.042 \mathrm{e}-04 \backslash \mathrm{Dipole}=-0.000$ $1977,-0.0000288,-0.0000234 \backslash P G=C 01[X(\mathrm{C} 8 \mathrm{H} 18)] \backslash \backslash @$

\section{$\mathrm{P}\left(\mathrm{CH}_{3}\right)_{4} \mathrm{P}\left(\mathrm{CH}_{3}\right)_{4}$}

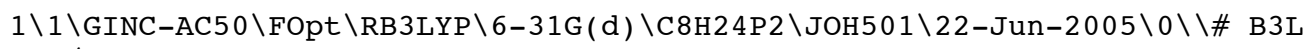
YP/6-31G* INT (GRID=ULTRAFINE) OPT FREQ=NORAMAN MAXDISK=671088640 IOP ( 1 $/ 8=10) \backslash \backslash$ me4ppme4_3 opt freq b3lyp $\backslash \backslash 0,1 \backslash P,-1.2401693218,0.0006070606,-0$ $.0034992034 \backslash \mathrm{P}, 1 . \overline{2} 398745521,-0.0009366644,0.0019507923 \backslash \mathrm{C},-1.323447886,1$ $.9149465696,-0.0072044145 \backslash \mathrm{C},-1.3335081843,-0.9438896247,1.6605593091 \backslash \mathrm{C}$ $,-1.317925035,-0.9690674411,-1.6556163825 \backslash C, 1.3247911111,0.9728110648$, $1.6492403061 \backslash \mathrm{C}, 1.3253934133,0.9389734674,-1.6678062533 \backslash \mathrm{C}, 1.3248140604$, $-1.9149414576,0.0373617558 \backslash \mathrm{H},-1.8935367756,2.2395491599,-0.8837208209 \backslash$ $\mathrm{H},-1.9098283625,-0.3437404795,2.3720943454 \backslash \mathrm{H},-1.8999282821,-1.88295544$ $46,-1.4988245379 \backslash \mathrm{H}, 1.8570152163,1.912694398,1.4701886276 \backslash \mathrm{H}, 1.861565522$ $8,0.3149539403,-2.3902096697 \backslash \mathrm{H}, 1.8557265405,-2.2208572417,0.9447447795$ $\backslash \mathrm{H},-0.3432744497,2.3955910355,-0.0188346835 \backslash \mathrm{H},-1.8724410778,2.24001417$ $05,0.8824860798 \backslash \mathrm{H},-1.8798405432,-1.878910921,1.4991705683 \backslash \mathrm{H},-0.3560936$ $604,-1.1706470834,2.0907637811 \backslash \mathrm{H},-0.3359090657,-1.2319051304,-2.053950$ $6056 \backslash \mathrm{H},-1.8521861352,-0.359551978,-2.3916364473 \backslash \mathrm{H}, 1.9112436463,0.39312$ $5028,2.369292531 \backslash \mathrm{H}, 0.3451688482,1.1930658527,2.0778918647 \backslash \mathrm{H}, 0.34576207$ $79,1.1959958155,-2.0753692683 \backslash \mathrm{H}, 1.9083456508,1.8545663543,-1.525386911$ $1 \backslash \mathrm{H}, 1.9122261932,-2.2578745094,-0.8203816156 \backslash \mathrm{H}, 0.344917277,-2.39593047$ $27,0.0172168327 \backslash \mathrm{C}, 3.2151199675,0.0004388804,-0.0005750354 \backslash \mathrm{C},-3.2145829$ $285,0.0011591161,-0.0140763488 \backslash \mathrm{H},-3.6134196975,-1.0208832186,0.0102223$ $664 \backslash \mathrm{H},-3.6186509987,0.535372425,0.855138283 \backslash \mathrm{H},-3.6078697965,0.48887601$ $69,-0.9150383034 \backslash \mathrm{H}, 3.6124849538,-0.4919159801,-0.8972712257 \backslash \mathrm{H}, 3.613464$ $3613,1.0227840559,0.020081121 \backslash \mathrm{H}, 3.6155529919,-0.5290551857,0.873261456$ $\backslash \backslash$ Version=IA6 4L-G03RevC. 02 \State $=1-A \backslash H F=-1001.8686003 \backslash R M S D=5.826 e-09 \backslash R$ $\mathrm{MSF}=5.400 \mathrm{e}-05 \backslash \mathrm{Dipole}=-0.0027113,-0.0005767,0.0067197 \backslash \mathrm{PG}=\mathrm{C} 01 \quad[\mathrm{X}(\mathrm{C} 8 \mathrm{H} 24 \mathrm{P} 2$ ) $] \backslash \backslash a$ 